\title{
Large-scale weakly nonlinear perturbations of convective magnetic dynamos in a rotating layer
}

\author{
R. Chertovskih ${ }^{\mathrm{a}}$, V. Zheligovsky ${ }^{\mathrm{b}}$ \\ ${ }^{a}$ Institute of Aeronautical Technology, \\ Praça Marechal Eduardo Gomes 50, Vila das Acácias, 12228-900 São José dos Campos, SP - Brazil \\ ${ }^{b}$ Institute of Earthquake Prediction Theory and Mathematical Geophysics, Russian Ac. Sci., \\ 84/32 Profsoyuznaya St, 117997 Moscow, Russian Federation
}

\begin{abstract}
We present a new mechanism for generation of large-scale magnetic field by thermal convection which does not involve the $\alpha$-effect. We consider weakly nonlinear perturbations of space-periodic steady convective magnetic dynamos in a rotating layer that were identified in our previous work. The perturbations have a spatial scale in the horizontal direction that is much larger than the period of the perturbed convective magnetohydrodynamic state. Following the formalism of the multiscale stability theory, we have derived the system of amplitude equations governing the evolution of the leading terms in the expansion of the perturbations in power series in the scale ratio. This asymptotic analysis is more involved than in the cases considered earlier, because the kernel of the operator of linearisation has zero-mean neutral modes whose origin lies in the spatial invariance of the perturbed regime, the operator reduced on the generalised kernel has two Jordan normal form blocks of size two, and simplifying symmetries of the perturbed state are now missing. Numerical results for the amplitude equations show that a large-scale perturbation, periodic in slow horizontal variable, either converges to a short-scale neutral stability mode with amplitudes tending to constant values, or it blows up at a finite slow time.
\end{abstract}

\section{Introduction}

A fundamental problem in astrophysics is to understand the sources of magnetic fields that are featured by many astrophysical bodies such as the Sun and the Earth. It is generally accepted that the magnetic fields are generated by hydromagnetic processes in the melted or fluid-like interiors of the bodies. This idea goes back to J. Larmor [15, 16]. It is widely believed that the so-called $\alpha$-effect plays a prominent role in such processes. Such a mechanism of magnetic field generation was first suggested by E. Parker [18, 19]. It relies on the frozenness of magnetic field into the conducting medium, when magnetic diffusion is negligible, implying that a small eddy in turbulent flow deforms the magnetic field force line into a loop. If this effect does not disappear when averaged over many loops, it gives rise to a mean electromotive force (e.m.f.), which can be parallel to the mean unperturbed magnetic field and can amplify the original mean field.

The theory of mean-field electrodynamics (MFE) is developed around a similar central idea [26, 13]: Suppose the flow and magnetic field are split into the mean and fluctuating parts, and the interaction of the fluctuating parts of the flow and the field yields a non-zero mean e.m.f. The MFE theory postulates that, at least in the case of magnetohydrodynamic (MHD) turbulence, the latter is related to the mean magnetic field (the coefficient of proportionality is traditionally denoted by $\alpha$, and, accordingly, the phenomenon is called the " $\alpha$-effect") combined, in some MFE models, with the spatial gradient of the mean field.

The MFE theory does not fully justify such relations. An insight into their mathematical roots is provided by the multiscale stability theory (MST). It treats an idealised case, in which turbu- 
lence is modelled by a small-scale laminar flow, periodic in space and time (see [33]). In contrast with MFE, mathematically rigorous results are then obtained by applying the asymptotic theory of PDE homogenisation to the problem of linear or weakly nonlinear stability of small-scale states. Purely hydrodynamic [8, 11] perturbations, the kinematic dynamo problem [14, 35, 34] (which is an instance of the general linear MHD stability problem, where the perturbed state is amagnetic, the flow and magnetic components of the perturbation therefore decouple, and one focuses on the magnetic perturbation), and full perturbations of forced MHD or convective MHD states [1, 29 31] (see also [33], Chap. 6-9) were considered. Perturbations are supposed to involve spatial and temporal scales that are much larger than the respective periods of the perturbed states. The perturbations are linear combinations of amplitude-modulated small-scale modes (i.e., eigenfunctions of the linearisation whose periods coincide with those of the perturbed states) associated with the same eigenvalue. The coefficients depend exclusively on slow temporal and spatial variables. They are usually called amplitudes, and the equations governing their dynamics are called amplitude equations. The evolution of such large-scale perturbations is essentially controlled by the associated eigenvalue of the constituting small-scale modes — only neutral (belonging to the kernel of linearisation) small-scale modes can instigate instability in the presence of large scales; consequently, MST usually focuses on large-scale amplitude modulation of neutral modes. Since typically small-scale neutral modes have non-zero means, amplitude equations for large-scale perturbations of forced MHD states (convective or not) are mean-field equations similar to those considered in MFE theory. However, as we will see, this is not always the case: translation-invariant physical systems, such as free thermal hydromagnetic convection in a horizontal layer investigated in the present paper, possess zero-mean neutral small-scale stability modes. Consequently, the mean-field description of the dynamics of large-scale perturbations of such physical systems is inadequate.

We intend to carry out a detailed investigation of stability to large-scale weakly nonlinear perturbations of the steady and time-periodic regimes of magnetic field generation by free thermal convection of rotating electrically conducting fluid in a horizontal layer, that were determined numerically in 7] and [32]. Here we consider space-periodic convective MHD steady states constituting the branch $S_{8}^{R 1}$ [7]. The group of symmetries of this steady state is generated by the symmetry about the vertical axis, $x_{3}$, (note that this is not axisymmetry, see section 2]) [7, 33] and the superposition of reflection about the midplane with translation by half a period in the horizontal direction $x_{1}$. This group of symmetries is smaller than other ones, typical for the parameter values considered ibid., and the system of amplitude equations, derived in [33], is inapplicable for large-scale perturbations of states having this group. The symmetry about a vertical axis implies that the steady state does not possess the $\alpha$-effect. The large-scale dynamics is due to the interplay of the combined eddy diffusivity and eddy advection. We find that their interaction cannot sustain stationary generation of large-scale magnetic field: a large-scale perturbation from the class which is described by the multiscale formalism either converges to a short-scale neutral stability mode, or it blows up at a finite time.

The paper is organised as follows. In section 2 we derive the system of amplitude equations for steady states of free hydromagnetic convection, which have the same group of symmetries as those comprising the branch $\mathrm{S}_{8}^{\mathrm{R} 1}$. In section 3 we present results of numerical analysis of the system of amplitude equations for several states belonging to this branch. Finally, we make remarks triggered by our investigation.

\section{The multiscale formalism for large-scale perturbations of free hydromagnetic convection}

In this section we derive amplitude equations for large-scale perturbations of small-scale steady

free hydromagnetic convection in a horizontal layer of electrically conducting fluid rotating about 
the vertical axis. A field, depending only on the fast spatial variables, $\mathbf{x}$, and time, $t$, is called small-scale; if, in addition, the field depends on the slow horizontal spatial variables, $\mathbf{X}=\varepsilon\left(x_{1}, x_{2}\right)$ and on the slow time, $T=\varepsilon^{s} t$, where $s \geq 1$, it is called large-scale. We will use asymptotic methods that are standard within the MST approach. We assume that the perturbed state is symmetric about the vertical axis and has equal periods $\mathscr{P}$ in $x_{1}$ and $x_{2}$; further assumptions are introduced where they become relevant.

A three-dimensional field $\mathbf{f}$ is called symmetric about a vertical axis passing through point $\left(a_{1}, a_{2}, 0\right)$ when the following conditions are satisfied [7, 33]:

$$
\begin{aligned}
& f_{1}\left(a_{1}-x_{1}, a_{2}-x_{2}, x_{3}\right)=-f_{1}\left(a_{1}+x_{1}, a_{2}+x_{2}, x_{3}\right), \\
& f_{2}\left(a_{1}-x_{1}, a_{2}-x_{2}, x_{3}\right)=-f_{2}\left(a_{1}+x_{1}, a_{2}+x_{2}, x_{3}\right), \\
& f_{3}\left(a_{1}-x_{1}, a_{2}-x_{2}, x_{3}\right)=f_{3}\left(a_{1}+x_{1}, a_{2}+x_{2}, x_{3}\right) .
\end{aligned}
$$

In particular, this symmetry implies that the flow is vertical everywhere on the axis. Together with the $\mathscr{P}$-periodicity in the horizontal directions, the symmetry about the Cartesian axis $x_{3}$ implies that the field is also symmetric about vertical axes through points $\mathscr{P} \mathbf{n} / 2$, where $\mathbf{n}=\left(n_{1}, n_{2}, 0\right)$ has integer components.

\subsection{Small-scale convective hydromagnetic steady states and their perturbations}

The state, whose stability we examine, is governed by the Navier-Stokes, magnetic induction and heat transfer equations. In the coordinate system, co-rotating with the fluid layer about the axis $x_{3}$, they are [6]

$$
\begin{aligned}
\partial \mathbf{V} / \partial t= & \nu \nabla^{2} \mathbf{V}+\mathbf{V} \times(\nabla \times \mathbf{V})-\mathbf{H} \times(\nabla \times \mathbf{H})+\beta \Theta \mathbf{e}_{3}+\tau \mathbf{V} \times \mathbf{e}_{3}-\nabla P \\
\partial \mathbf{H} / \partial t= & \eta \nabla^{2} \mathbf{H}+\nabla \times(\mathbf{V} \times \mathbf{H}) \\
\partial \Theta / \partial t= & \kappa \nabla^{2} \Theta-(\mathbf{V} \cdot \nabla) \Theta+\delta V_{3} \\
& \nabla \cdot \mathbf{V}=\nabla \cdot \mathbf{H}=0
\end{aligned}
$$

Here $\mathbf{V}$ is the flow velocity, $\mathbf{H}$ magnetic field, $\Theta=\vartheta-\vartheta_{1}+\delta x_{3}$ the difference between the temperature, $\vartheta$, and the steady state linear profile, $\vartheta_{1}$ and $\vartheta_{2}$ prescribed temperatures at the upper and lower horizontal boundaries $x_{3}=0$ and 1 , respectively, $\delta=\vartheta_{1}-\vartheta_{2}>0$ and $\tau=\sqrt{\mathrm{Ta}}$. The Navier-Stokes equation (11) involves buoyancy, Coriolis and Lorentz forces. No external body forces, heat or electric sources are present, i.e., there are no source terms in equations (10)-(41); in this case, we call convection free. Since the small-scale state $\mathbf{W}=(\mathbf{V}, \mathbf{H}, \Theta)$ is supposed to be steady, the time derivatives in the l.h.s. of (1)-(3) vanish.

Equations (1) -(4) are invariant with respect to the position of the axis of rotation, i.e., the equations are the same for any vertical axis of rotation. In an inertial (e.g., steady) coordinate system, a periodic arrangement of rotating convective cells, with the pressure periodic in horizontal directions, is impossible. Periodicity in $x_{1}$ and $x_{2}$ becomes possible after the pressure is corrected by $\tau^{2} \rho^{2} / 8$, where $\rho$ is the distance to the axis of rotation (see [6]); this term represents the centripetal force preserving the arrangement of cells (regarded as rigid bodies) rotating with constant angular velocity $\tau / 2$. Clearly, rotation about a vertical axis is compatible with the symmetry about a vertical line; however, since the governing equations are invariant with respect to the position of the axis of rotation, the axis of rotation does not necessarily pass through the centre of a periodicity cell or coincides with the axis about which the flow is symmetric.

We consider horizontal boundaries of the fluid layer that are stress-free, ideally electrically conducting and kept at constant temperatures. This translates into the following equations:

$$
\begin{aligned}
\partial V_{1} / \partial x_{3}=\partial V_{2} / \partial x_{3}=V_{3} & =0, \\
\partial H_{1} / \partial x_{3}=\partial H_{2} / \partial x_{3}=H_{3} & =0, \\
\Theta & =0 .
\end{aligned}
$$


We define the mean over the fast spatial variables, $\langle\cdot\rangle$, and the fluctuating, $\{\cdot\}$, parts of a field $f$ :

$$
\langle f(\mathbf{X}, T, \mathbf{x}, t)\rangle \equiv \frac{1}{\mathscr{P}^{2}} \int_{-\mathscr{P} / 2}^{\mathscr{P} / 2} \int_{-\mathscr{P} / 2}^{\mathscr{P} / 2} \int_{0}^{1} f(\mathbf{X}, T, \mathbf{x}, t) \mathrm{d} \mathbf{x}, \quad\{f\} \equiv f-\langle f\rangle .
$$

Same definitions hold for a vector field $\mathbf{f}$, and we also define the mean of its horizontal part, $\langle\mathbf{f}\rangle_{h} \equiv\left\langle f_{1}\right\rangle \mathbf{e}_{1}+\left\langle f_{2}\right\rangle \mathbf{e}_{2}$, and the fluctuating part $\{\mathbf{f}\}_{h} \equiv \mathbf{f}-\langle\mathbf{f}\rangle_{h}$. Later (see section 2.2.1) it will become clear why these averaging procedures agree with the mathematical nature of the problem. We note that the Coriolis force can be reexpressed as $\tau\{\mathbf{V}\}_{h} \times \mathbf{e}_{3}$, since the constant horizontal force $\tau\langle\mathbf{V}\rangle_{h} \times \mathbf{e}_{3}$ is a gradient of a linear function of horizontal coordinates that modifies pressure.

Upon this modification, the operator of linearisation of equations (11)-(3) around the steady state $\mathbf{W}$, which we denote by $\mathfrak{L}=\left(\mathfrak{L}^{v}, \mathfrak{L}^{h}, \mathfrak{L}^{\theta}\right)$, takes the form

$$
\begin{aligned}
\mathfrak{L}^{v}(\mathbf{v}, \mathbf{h}, \theta)= & -\partial \mathbf{v} / \partial t+\nu \nabla^{2} \mathbf{v}+\mathbf{V} \times(\nabla \times \mathbf{v})+\mathbf{v} \times(\nabla \times \mathbf{V}) \\
& -\mathbf{H} \times(\nabla \times \mathbf{h})-\mathbf{h} \times(\nabla \times \mathbf{H})+\beta \theta \mathbf{e}_{3}+\tau\{\mathbf{v}\}_{h} \times \mathbf{e}_{3}-\nabla p, \\
\mathfrak{L}^{h}(\mathbf{v}, \mathbf{h}, \theta)= & -\partial \mathbf{h} / \partial t+\eta \nabla^{2} \mathbf{h}+\nabla \times(\mathbf{V} \times \mathbf{h}+\mathbf{v} \times \mathbf{H}), \\
\mathfrak{L}^{\theta}(\mathbf{v}, \mathbf{h}, \theta)= & -\partial \theta / \partial t+\kappa \nabla^{2} \theta-(\mathbf{V} \cdot \nabla) \theta-(\mathbf{v} \cdot \nabla) \Theta+\delta v_{3} .
\end{aligned}
$$

We consider a perturbation of the steady state $\mathbf{W}$, whose amplitude is $\mathrm{O}(\varepsilon)$. The perturbed state, $(\mathbf{V}+\varepsilon \mathbf{v}, \mathbf{H}+\varepsilon \mathbf{h}, \Theta+\varepsilon \theta)$, satisfies equations (1)-(4), whereby

$$
\begin{aligned}
\mathfrak{L}^{v}(\mathbf{v}, \mathbf{h}, \theta) & =-\varepsilon(\mathbf{v} \times(\nabla \times \mathbf{v})-\mathbf{h} \times(\nabla \times \mathbf{h}))+\nabla p \\
\mathfrak{L}^{h}(\mathbf{v}, \mathbf{h}, \theta) & =-\varepsilon \nabla \times(\mathbf{v} \times \mathbf{h}), \\
\mathfrak{L}^{\theta}(\mathbf{v}, \mathbf{h}, \theta) & =\varepsilon(\mathbf{v} \cdot \nabla) \theta \\
\nabla \cdot \mathbf{v} & =\nabla \cdot \mathbf{h}=0
\end{aligned}
$$

Following the recipes of the homogenisation techniques, we now assume that the perturbations depend on the slow horizontal spatial variables $\mathbf{X}=\varepsilon\left(x_{1}, x_{2}\right)$ and on the slow time, $T=\varepsilon^{s} t$, where $s \geq 1$, and expand the perturbation in a power series in the small scale ratio $\varepsilon$ :

$$
\begin{aligned}
\mathbf{v} & =\sum_{n=0}^{\infty} \mathbf{v}_{n}(\mathbf{X}, T, \mathbf{x}, t) \varepsilon^{n}, \\
\mathbf{h} & =\sum_{n=0}^{\infty} \mathbf{h}_{n}(\mathbf{X}, T, \mathbf{x}, t) \varepsilon^{n}, \\
\theta & =\sum_{n=0}^{\infty} \theta_{n}(\mathbf{X}, T, \mathbf{x}, t) \varepsilon^{n} .
\end{aligned}
$$

Upon substituting these series into the governing equations (8)-(11), we obtain a hierarchy of systems of equations. In principle, it can be solved at any order, and thus complete series (12) -(14) can be reconstructed. In this paper we are only interested in the leading-order terms and therefore will only consider the first 3 systems in the hierarchy.

The solenoidality conditions (11) at order $\varepsilon^{n}$ yield equations, whose mean and fluctuating parts for any $n \geq 0$ are

$$
\begin{gathered}
\nabla_{\mathbf{X}} \cdot\left\langle\mathbf{v}_{n}\right\rangle_{h}=0 \\
\nabla_{\mathbf{X}} \cdot\left\langle\mathbf{h}_{n}\right\rangle_{h}=0 \\
\nabla_{\mathbf{X}} \cdot \mathbf{v}_{n}+\nabla_{\mathbf{X}} \cdot\left\{\mathbf{v}_{n-1}\right\}_{h}=0 \\
\nabla_{\mathbf{x}} \cdot \mathbf{h}_{n}+\nabla_{\mathbf{X}} \cdot\left\{\mathbf{h}_{n-1}\right\}_{h}=0
\end{gathered}
$$


(any term of expansions with a negative index is zero by definition). We can now comment on the modified form of the Coriolis force term in the operator $\mathfrak{L}^{v}$ in (8): By virtue of solenoidality (15) of the mean field, we can introduce a stream function, $\psi$, whereby $\langle\mathbf{v}\rangle_{h}=\left(-\partial \psi / \partial X_{2}, \partial \psi / \partial X_{1}, 0\right)$. Thus, the difference between the original, $\tau \mathbf{v} \times \mathbf{e}_{3}$, and modified, $\tau\{\mathbf{v}\}_{h} \times \mathbf{e}_{3}$, forms is equal to $\tau\langle\mathbf{v}\rangle_{h} \times \mathbf{e}_{3}=\tau \nabla_{\mathbf{X}} \psi$, which is compensated by modifying the pressure perturbation, $p$. So, although now the flow perturbation, $\mathbf{v}$, depends on the slow spatial variables, the operator of linearisation, $\mathfrak{L}^{v}$, of the Navier-Stokes equation (1) can still be used in the form (8)).

We assume henceforth that the pressure perturbation $p$ is $\mathscr{P}$-periodic in the fast horizontal variables.

\section{2. $O r d e r \varepsilon^{0}$ equations}

This section is devoted to the study of the generalised kernel of the operator of linearisation $\mathfrak{L}$. We find that the operator acting on the generalised kernel has two Jordan normal form blocks of size two, and, consequently, the means of the flow and magnetic components of neutral modes of $\mathfrak{L}$, $\mathbf{S}_{k}$ for $k=1,2$, are related by a matrix $\mathcal{U}$ of size two introduced below. The perturbed states are missing the symmetries which make this matrix vanish. As a result, it will turn out that because of the solenoidality in the slow variable of the spatially averaged perturbations of the form (12)-(14), the latter can be described by a self-consistent system of amplitude equations only if they depend on a single spatial variable $Y=\mathbf{q} \cdot \mathbf{X}$, where a unit vector $\mathbf{q}$ is normal to an eigenvector of $\mathcal{U}$.

\subsubsection{The Jordan normal form of the operator of linearisation}

in the invariant subspace associated with the zero eigenvalue

The first system of equations in the hierarchy is obtained at order $\varepsilon^{0}$ :

$$
\mathfrak{L}\left(\mathbf{v}_{0}, \mathbf{b}_{0}, \theta_{0}\right)=0, \quad \nabla_{\mathbf{x}} \cdot \mathbf{v}_{0}=\nabla_{\mathbf{x}} \cdot \mathbf{b}_{0}=0,
$$

i.e., the field $\left(\mathbf{v}_{0}, \mathbf{b}_{0}, \theta_{0}\right)$ belongs to the kernel of the operator of linearisation, $\mathfrak{L}$; it must satisfy the requirements for perturbation, i.e., the solenoidality conditions for $\mathbf{v}$ and $\mathbf{h}, \mathscr{P}$-periodicity in the fast horizontal variables and boundary conditions (15)-(7).

As usual, we derive the adjoint operator $\mathfrak{L}^{*}=\left(\mathfrak{L}^{* v}, \mathfrak{L}^{* h}, \mathfrak{L}^{* \theta}\right)$ in the Lebesgue space $\mathrm{L}_{2}$ of solenoidal vector fields by using vector analysis identities and, essentially, integrating by parts the l.h.s. of the defining equation

$$
\begin{aligned}
\mathfrak{L} \mathbf{w} \cdot \mathbf{w}^{*}=\mathbf{w} \cdot \mathfrak{L}^{*} \mathbf{w}^{*}: & \\
\mathfrak{L}^{* v}(\mathbf{v}, \mathbf{h}, \theta)= & \partial \mathbf{v} / \partial t+\nu \nabla^{2} \mathbf{v}-\nabla \times(\mathbf{V} \times \mathbf{v}) \\
& +\mathfrak{P}\left(\mathbf{H} \times(\nabla \times \mathbf{h})-\mathbf{v} \times(\nabla \times \mathbf{V})+\delta \theta \mathbf{e}_{3}-\tau\{\mathbf{v}\} \times \mathbf{e}_{3}+\Theta \nabla \theta\right), \\
\mathfrak{L}^{* h}(\mathbf{v}, \mathbf{h}, \theta)= & \partial \mathbf{h} / \partial t+\eta \nabla^{2} \mathbf{h}+\nabla \times(\mathbf{H} \times \mathbf{v})+\mathfrak{P}(\mathbf{v} \times(\nabla \times \mathbf{H})-\mathbf{V} \times(\nabla \times \mathbf{h})), \\
\mathfrak{L}^{* \theta}(\mathbf{v}, \mathbf{h}, \theta)= & \partial \theta / \partial t+\kappa \nabla^{2} \theta+(\mathbf{V} \cdot \nabla) \theta+\beta v_{3} .
\end{aligned}
$$

Here $\mathfrak{P}$ is the projection onto the subspace of solenoidal vector fields satisfying the requirements for perturbation.

We assume henceforth that the small-scale perturbed steady state $\mathbf{W}=(\mathbf{V}, \mathbf{H}, \Theta)$ is stable to small-scale perturbations, i.e., real parts of all eigenvalues of $\mathfrak{L}$ are non-positive. It is then natural to assume that the evolution of large-scale perturbations of $\mathbf{W}$ is examined after all the fast-time transients have decayed. Since the perturbed state is steady, we will assume that the perturbations do not depend on the fast time, and hence the derivatives in $t$ in the definitions of the operators $\mathfrak{L}$ and $\mathfrak{L}^{*}$ will be omitted. (It would be necessary to preserve them when investigating large-scale stability of time-periodic states.) 
Since $\mathfrak{L}^{*}\left(\mathbf{e}_{k}, 0,0\right)=\mathfrak{L}^{*}\left(0, \mathbf{e}_{k}, 0\right)=0$ for $k=1,2$, the kernel of the operator $\mathfrak{L}$ is at least fourdimensional. Differentiating (11)-(4) in $x_{k}$ for $k=1,2$, we find that

$$
\mathbf{S}_{k+2}=\partial \mathbf{W} / \partial x_{k}
$$

belong to the kernel of $\mathfrak{L}$. Furthermore,

$$
\mathfrak{L}\left(\mathbf{e}_{k}, 0,0\right)=-\mathbf{S}_{k+2} \in \operatorname{ker} \mathfrak{L}
$$

therefore, the Jordan normal form of $\mathfrak{L}$ involves two blocks of size 2 associated with the eigenvalue zero, and $\mathfrak{L}$ has at least 6 eigenfields associated with this eigenvalue, at least two of which are generalised. Henceforth, we assume the generic case where zero is a 6-fold (generalised) eigenvalue of $\mathfrak{L}$. Clearly, $\left\langle\mathfrak{L}^{v}(\mathbf{w})\right\rangle_{h}=\left\langle\mathfrak{L}^{h}(\mathbf{w})\right\rangle_{h}=0$ for any $\mathbf{w}=(\mathbf{v}, \mathbf{h}, \theta)$ satisfying the requirements for perturbation. Consequently, it is simple to show that any eigenfield, a generalised one or not, which has a non-zero mean horizontal part of the flow and/or magnetic component, is associated with the zero eigenvalue. Such eigenfields exist, because eigenfields of the elliptic operator $\mathfrak{L}$ constitute a complete basis in the Lebesgue space $\mathrm{L}_{2}$; hence, the kernel of $\mathfrak{L}$ involves the fields $\mathbf{S}_{k}=\left(\mathbf{S}_{k}^{v}, \mathbf{S}_{k}^{h}, S_{k}^{\theta}\right)$ for $k=1,2$, satisfying the requirements for perturbation and such that

$$
\mathfrak{L S}_{k}=0, \quad \mathbf{S}_{k}^{v}=\left\{\mathbf{S}_{k}^{v}\right\}_{h}+\sum_{m=1}^{2} u_{m k} \mathbf{e}_{m}, \quad \mathbf{S}_{k}^{h}=\left\{\mathbf{S}_{k}^{h}\right\}_{h}+\mathbf{e}_{k}
$$

The first auxiliary problem is to find the fields $\mathbf{S}_{k}$ and the matrix $\mathcal{U}=\left[\begin{array}{ll}u_{11} & u_{12} \\ u_{21} & u_{22}\end{array}\right]$. Since normal Jordan forms of an operator and its adjoint coincide, there exist two generalised eigenfields $\mathbf{S}_{n}^{*}=\left(\mathbf{S}_{n}^{* v}, \mathbf{S}_{n}^{* h}, S_{n}^{* \theta}\right), n=1,2$, associated with the eigenvalue zero of $\mathfrak{L}^{*}$, i.e., such that

$$
\mathfrak{L}^{*} \mathbf{S}_{n}^{*}=\left(\sum_{m=1}^{2} u_{m n}^{\prime} \mathbf{e}_{m}, \mathbf{e}_{n}, 0\right), \quad\left\langle\mathbf{S}_{n}^{* v}\right\rangle_{h}=\left\langle\mathbf{S}_{n}^{* h}\right\rangle_{h}=0, \quad \nabla_{\mathbf{x}} \cdot \mathbf{S}_{n}^{* v}=\nabla_{\mathbf{x}} \cdot \mathbf{S}_{n}^{* h}=0
$$

and also satisfying the requirements for perturbation. It is straightforward to demonstrate that

$$
\left[\begin{array}{ll}
u_{11}^{\prime} & u_{12}^{\prime} \\
u_{21}^{\prime} & u_{22}^{\prime}
\end{array}\right]=-\left(\mathcal{U}^{t}\right)^{-1}
$$

where the superscript $t$ denotes the transpose of a matrix.

By linearity of the system of order $\varepsilon^{0}$ equations (19), its solution is

$$
\mathbf{w}_{0}=\left(\mathbf{v}_{0}, \mathbf{h}_{0}, \theta_{0}\right)=\sum_{k=1}^{4} c_{0 k}(\mathbf{X}, T) \mathbf{S}_{k}(\mathbf{x}), \quad p_{0}=\sum_{k=1}^{4} c_{0 k}(\mathbf{X}, T) S_{k}^{p}(\mathbf{x}),
$$

where $p_{0}$ and $S_{k}^{p}$ denote the gradient potentials arising in the equations $\mathfrak{L}^{v} \mathbf{w}_{0}=0$ and $\mathfrak{L}^{v} \mathbf{S}_{k}=0$, respectively. The horizontal mean of the magnetic component of the first relation in (25) reduces to $c_{0 k}=\left\langle h_{0, k}\right\rangle$ for $k=1,2$, and hence $\left\langle\mathbf{v}_{0}\right\rangle_{h}=\mathcal{U}\left\langle\mathbf{h}_{0}\right\rangle_{h}$.

The solvability condition for a problem $\mathfrak{L} \mathbf{w}=\left(\mathbf{f}^{v}, \mathbf{f}^{h}, f^{\theta}\right)$ consists of orthogonality of the r.h.s. to the kernel of the adjoint $\mathfrak{L}^{*}$. Since the kernel of $\mathfrak{L}^{*}$ is comprised of constant vectors in the horizontal flow and magnetic components, the solvability condition reduces to $\left\langle\mathbf{f}^{v}\right\rangle_{h}=\left\langle\mathbf{f}^{h}\right\rangle_{h}=0$. It is straightforward to establish that this condition is necessary; that it is sufficient follows from the Fredholm alternative theorem applied to the problem $\left(\nabla^{2}\right)^{-1} \mathfrak{L} \mathbf{w}=\left(\nabla^{2}\right)^{-1}\left(\mathbf{f}^{v}, \mathbf{f}^{h}, f^{\theta}\right)$ (the proof is essentially the same as in section 3.2 in [33]). It is due to this form of the solvability condition that spatial averaging over the periodicity domain is the only appropriate kind of averaging in the problem under investigation. 


\subsubsection{Solenoidality of the mean fields}

Here we discuss how to satisfy the solenoidality conditions (15)-(16). We assume that amplitudes $c_{0 k}$ are space-periodic in the slow variables $\mathbf{X}=\left(X_{1}, X_{2}\right)$ and expand the amplitudes in Fourier series, which it is convenient to express in the form

$$
\left\langle\mathbf{v}_{0}\right\rangle_{h}=\sum_{k,|\mathbf{q}|=\mathbf{1}}\left(v_{1, k \mathbf{q}} \mathbf{e}_{1}+v_{2, k \mathbf{q}} \mathbf{e}_{2}\right) \mathrm{e}^{\mathrm{i} k \mathbf{q} \cdot \mathbf{X}}, \quad\left\langle\mathbf{h}_{0}\right\rangle_{h}=\sum_{k,|\mathbf{q}|=\mathbf{1}}\left(h_{1, k \mathbf{q}} \mathbf{e}_{1}+h_{2, k \mathbf{q}} \mathbf{e}_{2}\right) \mathrm{e}^{\mathrm{i} k \mathbf{q} \cdot \mathbf{X}}
$$

and consider the conditions independently for each unit wave vector $\mathbf{q}=\left(q_{1}, q_{2}\right)$.

Solenoidality of $\left\langle\mathbf{h}_{0}\right\rangle_{h}$ then requires $\left(h_{1, k \mathbf{q}}, h_{2, k \mathbf{q}}\right)=C_{k \mathbf{q}} \mathbf{q}^{\perp}$, where $\mathbf{q}^{\perp}=\left(q_{2},-q_{1}\right)$, and hence solenoidality of $\left[\begin{array}{l}v_{1, k \mathbf{q}} \\ v_{2, k \mathbf{q}}\end{array}\right]=\mathcal{U}\left[\begin{array}{l}h_{1, k \mathbf{q}} \\ h_{2, k \mathbf{q}}\end{array}\right]$ is equivalent to the orthogonality condition $\mathcal{U} \mathbf{q}^{\perp} \cdot \mathbf{q}=0$. It implies $\mathbf{q}^{\perp} \| \mathcal{U} \mathbf{q}^{\perp}$; thus, $\mathbf{q}^{\perp}$ is an eigenvector of $\mathcal{U}$. We denote the associated eigenvalue by $\lambda=\mathbf{q}^{\perp} \cdot \mathcal{U} \mathbf{q}^{\perp}$. In the basis $\left\{\mathbf{q}^{\perp}, \mathbf{q}\right\}$, the matrix $\mathcal{U}$ is upper triangular; consequently, the wave vector $\mathbf{q}$ is an eigenvector of $\mathcal{U}^{t}$ associated with the second eigenvalue $\lambda^{\prime}=\mathbf{q} \cdot \mathcal{U} \mathbf{q}$.

Thus, by virtue of the solenoidality conditions the series (26) may involve only two wave vectors, $\mathbf{q}^{1}$ and $\mathbf{q}^{2}$, that are normal to eigenvectors of $\mathcal{U}$; hence $\left\langle\mathbf{v}_{0}\right\rangle_{h}$ and $\left\langle\mathbf{h}_{0}\right\rangle_{h}$ split into sums of vector fields, each depending on just one spatial variable, $Y_{1}=\mathbf{q}^{1} \cdot \mathbf{X}$ or $Y_{2}=\mathbf{q}^{2} \cdot \mathbf{X}$. We will see in section 2.4 that the system of amplitude equations for $c_{0 k}$ is nonlinear (cubic). Therefore, a weakly nonlinear perturbation depends on one spatial variable $Y=\mathbf{q} \cdot \mathbf{X}$ such that $\mathbf{q}^{\perp}$ is an eigenvector of $\mathcal{U}$ (otherwise, products of functions of $Y_{1}$ and $Y_{2}$ are generated by the nonlinearity of the governing equations for $c_{0 k}$ ). Perturbations with initial amplitudes that violate this onedimensionality restriction cannot thus be expanded in the series (12)-(14) and are not described by homogenised equations that we will derive. Arising for $\mathcal{U} \neq 0$, this is the main difference with the cases considered in [33]. Summarising,

$$
\left[\begin{array}{c}
\left\langle v_{0,1}\right\rangle \\
\left\langle v_{0,2}\right\rangle
\end{array}\right]=\lambda C(Y, T) \mathbf{q}^{\perp}, \quad\left[\begin{array}{c}
\left\langle h_{0,1}\right\rangle \\
\left\langle h_{0,2}\right\rangle
\end{array}\right]=C(Y, T) \mathbf{q}^{\perp}, \quad \mathcal{U} \mathbf{q}^{\perp}=\lambda \mathbf{q}^{\perp}, \quad Y=\mathbf{q} \cdot \mathbf{X} .
$$

We will henceforth use the same notation for the two-dimensional vector $\mathbf{q}$ and the threedimensional horizontal vector, whose horizontal component coincides with $\mathbf{q}$; similarly for $\mathbf{q}^{\perp}$.

\section{3. $O r d e r \varepsilon^{1}$ equations}

At order $\varepsilon^{1}$ we obtain the system of equations

$$
\begin{aligned}
\mathfrak{L}^{v}\left(\mathbf{v}_{1}, \mathbf{h}_{1}, \theta_{1}\right) & +2 \nu\left(\nabla_{\mathbf{x}} \cdot \nabla_{\mathbf{X}}\right) \mathbf{v}_{0}+\mathbf{V} \times\left(\nabla_{\mathbf{X}} \times \mathbf{v}_{0}\right)-\mathbf{H} \times\left(\nabla_{\mathbf{X}} \times \mathbf{h}_{0}\right) \\
& +\mathbf{v}_{0} \times\left(\nabla_{\mathbf{x}} \times \mathbf{v}_{0}\right)-\mathbf{h}_{0} \times\left(\nabla_{\mathbf{x}} \times \mathbf{h}_{0}\right)-\nabla_{\mathbf{X}} p_{0}=0, \\
\mathfrak{L}^{h}\left(\mathbf{v}_{1}, \mathbf{h}_{1}, \theta_{1}\right) & +2 \eta\left(\nabla_{\mathbf{x}} \cdot \nabla_{\mathbf{X}}\right) \mathbf{h}_{0}+\nabla_{\mathbf{X}} \times\left(\mathbf{v}_{0} \times \mathbf{H}+\mathbf{V} \times \mathbf{h}_{0}\right)+\nabla_{\mathbf{x}} \times\left(\mathbf{v}_{0} \times \mathbf{h}_{0}\right)=0, \\
\mathfrak{L}^{\theta}\left(\mathbf{v}_{1}, \mathbf{h}_{1}, \theta_{1}\right) & +2 \kappa\left(\nabla_{\mathbf{x}} \cdot \nabla_{\mathbf{X}}\right) \theta_{0}-\left(\mathbf{V} \cdot \nabla_{\mathbf{X}}\right) \theta_{0}-\left(\mathbf{v}_{0} \cdot \nabla_{\mathbf{x}}\right) \theta_{0}=0 .
\end{aligned}
$$

For the slow time $T=\varepsilon t$, these equations would also involve the derivatives of the respective fields in the slow time. The solvability condition then defines the operator of the significant combined $\alpha$-effect: it consists of spatial means of linear (in $\mathbf{w}_{0}$ ) terms in the horizontal components of the flow and magnetic equations. However, the perturbed state $\mathbf{W}=(\mathbf{V}, \mathbf{H}, \Theta)$ is supposed to be symmetric about the vertical axis, $x_{3}$. Consequently, solutions to the first auxiliary problems are antisymmetric, the r.h.s. of the equations are symmetric, and the $\alpha$-effect operator is zero: convective hydromagnetic states with such a symmetry lack any significant (i.e., responsible for the evolution of large-scale amplitudes) $\alpha$-effect. In the absence of significant $\alpha$-effect, we have to 
switch to the slow time $T=\varepsilon^{2} t$. Since the solvability condition is verified, a solution to this system exists and, by linearity, takes the form

$$
\left(\begin{array}{c}
\mathbf{v}_{1} \\
\mathbf{h}_{1} \\
\theta_{1}
\end{array}\right)=\sum_{k=1}^{4}\left(c_{1 k} \mathbf{S}_{k}+\sum_{m=1}^{2} \mathbf{G}_{m k} \frac{\partial c_{0 k}}{\partial X_{m}}+\sum_{m=1}^{4} \mathbf{Q}_{m k} c_{0 k} c_{0 m}\right),
$$

where $\mathbf{G}_{m k}(\mathbf{x}, t)$ for $k=1, \ldots, 4$ and $m=1,2$ are solutions to the second auxiliary problem:

$$
\begin{aligned}
\mathfrak{L}^{v} \mathbf{G}_{m k}= & -2 \nu \partial \mathbf{S}_{k}^{v} / \partial x_{m}-\mathbf{V} \times\left(\mathbf{e}_{m} \times \mathbf{S}_{k}^{v}\right)+\mathbf{H} \times\left(\mathbf{e}_{m} \times \mathbf{S}_{k}^{h}\right)+S_{k}^{p} \mathbf{e}_{m}, \\
\mathfrak{L}^{h} \mathbf{G}_{m k}= & -2 \eta \partial \mathbf{S}_{k}^{h} / \partial x_{m}-\mathbf{e}_{m} \times\left(\mathbf{V} \times \mathbf{S}_{k}^{h}+\mathbf{S}_{k}^{v} \times \mathbf{H}\right), \\
\mathfrak{L}^{\theta} \mathbf{G}_{m k}= & -2 \kappa \partial S_{k}^{\theta} / \partial x_{m}+V_{m} S_{k}^{\theta}, \\
& \nabla \cdot \mathbf{G}_{m k}^{v}=-\left\{S_{k, m}^{v}\right\}, \quad \nabla \cdot \mathbf{G}_{m k}^{h}=-\left\{S_{k, m}^{h}\right\}
\end{aligned}
$$

and $\mathbf{Q}_{m k}(\mathbf{x}, t)$ for $m, k=1, \ldots, 4$ are solutions to the third auxiliary problem:

$$
\begin{aligned}
2 \mathfrak{L}^{v} \mathbf{Q}_{m k} & =-\mathbf{S}_{k}^{v} \times\left(\nabla \times \mathbf{S}_{m}^{v}\right)+\mathbf{S}_{k}^{h} \times\left(\nabla \times \mathbf{S}_{m}^{h}\right)-\mathbf{S}_{m}^{v} \times\left(\nabla \times \mathbf{S}_{k}^{v}\right)+\mathbf{S}_{m}^{h} \times\left(\nabla \times \mathbf{S}_{k}^{h}\right), \\
2 \mathfrak{L}^{h} \mathbf{Q}_{m k} & =-\nabla \times\left(\mathbf{S}_{k}^{v} \times \mathbf{S}_{m}^{h}+\mathbf{S}_{m}^{v} \times \mathbf{S}_{k}^{h}\right), \\
2 \mathfrak{L}^{\theta} \mathbf{Q}_{m k} & =\left(\mathbf{S}_{k}^{v} \cdot \nabla\right) S_{m}^{\theta}+\left(\mathbf{S}_{m}^{v} \cdot \nabla\right) S_{k}^{\theta}, \\
\nabla \cdot \mathbf{Q}_{m k}^{v} & =\nabla \cdot \mathbf{Q}_{m k}^{h}=0
\end{aligned}
$$

(for the symmetrised last sum in (31)). The potential $p_{1}$ in (28) can be expressed in terms of the gradient potentials $G_{k}^{p}$ and $Q_{k}^{p}$ arising in (32) and (36), where the flow components $\mathbf{G}_{m k}^{v}$ and $\mathbf{Q}_{m k}^{v}$ satisfy relations (35) and (39), respectively:

$$
p_{1}=\sum_{k=1}^{4}\left(c_{1 k} S_{k}^{p}+\sum_{m=1}^{2} G_{m k}^{p} \frac{\partial c_{0 k}}{\partial X_{m}}+\sum_{m=1}^{4} Q_{m k}^{p} c_{0 k} c_{0 m}\right), \quad\left\langle p_{1}\right\rangle=0 .
$$

For the same symmetry reasons as for the full equations (28)-(30), the r.h.s. of the equations in formulations of the second and third auxiliary problems are symmetric, and hence the solvability conditions for them are satisfied. Symmetric and antisymmetric fields constitute invariant subspaces of the operator of linearisation $\mathfrak{L}$, and thus $\mathbf{G}_{m k}$ and $\mathbf{Q}_{m k}$ are symmetric about the vertical axis.

Examining the system of equations for the difference $\mathbf{G}_{14}-\mathbf{G}_{23}$, it is straightforward (albeit tedious) to show that

$$
\mathbf{G}_{14}-\mathbf{G}_{23}=\left(\mathbf{V} \times \mathbf{e}_{3}, \mathbf{H} \times \mathbf{e}_{3}, 0\right) .
$$

Differentiating the equation $\mathfrak{L}\left(\mathbf{S}_{k}\right)=0$ in $x_{1}$ and $x_{2}$ and comparing the results with (36)-(39)), we find

$$
\partial \mathbf{S}_{k} / \partial x_{m-2}=2 \mathbf{Q}_{m k}=2 \mathbf{Q}_{k m},
$$

for any $k=1, \ldots, 4$ and $m=3,4$.

\subsection{Order $\varepsilon^{2}$ equations}

At order $\varepsilon^{2}$ we obtain the system of equations

$$
\begin{aligned}
\partial \mathbf{v}_{0} / \partial T= & \mathfrak{L}^{v}\left(\mathbf{v}_{2}, \mathbf{h}_{2}, \theta_{2}\right)+2 \nu\left(\nabla_{\mathbf{x}} \cdot \nabla_{\mathbf{X}}\right) \mathbf{v}_{1}+\nu \nabla_{\mathbf{X}}^{2} \mathbf{v}_{0} \\
& +\mathbf{V} \times\left(\nabla_{\mathbf{X}} \times \mathbf{v}_{1}\right)-\mathbf{H} \times\left(\nabla_{\mathbf{X}} \times \mathbf{h}_{1}\right)+\mathbf{v}_{0} \times\left(\nabla_{\mathbf{X}} \times \mathbf{v}_{0}+\nabla_{\mathbf{x}} \times \mathbf{v}_{1}\right) \\
& -\mathbf{h}_{0} \times\left(\nabla_{\mathbf{X}} \times \mathbf{h}_{0}+\nabla_{\mathbf{x}} \times \mathbf{h}_{1}\right)+\mathbf{v}_{1} \times\left(\nabla_{\mathbf{x}} \times \mathbf{v}_{0}\right)-\mathbf{h}_{1} \times\left(\nabla_{\mathbf{x}} \times \mathbf{h}_{0}\right)-\nabla_{\mathbf{X}} p_{1}, \\
\partial \mathbf{h}_{0} / \partial T= & \mathfrak{L}^{h}\left(\mathbf{v}_{2}, \mathbf{h}_{2}, \theta_{2}\right)+2 \eta\left(\nabla_{\mathbf{x}} \cdot \nabla_{\mathbf{X}}\right) \mathbf{h}_{1}+\eta \nabla_{\mathbf{X}}^{2} \mathbf{h}_{0} \\
& +\nabla_{\mathbf{X}} \times\left(\mathbf{v}_{1} \times \mathbf{H}+\mathbf{V} \times \mathbf{h}_{1}+\mathbf{v}_{0} \times \mathbf{h}_{0}\right)+\nabla_{\mathbf{x}} \times\left(\mathbf{v}_{0} \times \mathbf{h}_{1}+\mathbf{v}_{1} \times \mathbf{h}_{0}\right), \\
\partial \theta_{0} / \partial T= & \mathfrak{L}^{\theta}\left(\mathbf{v}_{2}, \mathbf{h}_{2}, \theta_{2}\right)+2 \kappa\left(\nabla_{\mathbf{x}} \cdot \nabla_{\mathbf{X}}\right) \theta_{1}+\kappa \nabla_{\mathbf{X}}^{2} \theta_{0} \\
& -\left(\mathbf{v}_{1} \cdot \nabla_{\mathbf{x}}\right) \theta_{0}-\left(\mathbf{v}_{0} \cdot \nabla_{\mathbf{x}}\right) \theta_{1}-\left(\mathbf{v}_{0} \cdot \nabla_{\mathbf{X}}\right) \theta_{0}-\left(\mathbf{V} \cdot \nabla_{\mathbf{X}}\right) \theta_{1} .
\end{aligned}
$$


Two equations for the evolution of amplitudes of large-scale perturbations are the solvability conditions for the system (42)-(44): orthogonality to the kernel of the adjoint operator, $\mathfrak{L}^{*}$, i.e., the requirement of vanishing of the horizontal means of the flow and magnetic equations. By (42),

$$
\frac{\partial\left\langle\mathbf{v}_{0}\right\rangle_{h}}{\partial T}=\nu \nabla_{\mathbf{X}}^{2}\left\langle\mathbf{v}_{0}\right\rangle_{h}+\sum_{j=1}^{2} \sum_{k=1}^{4}\left(\sum_{m=1}^{2} \mathbf{D}_{j m k}^{v} \frac{\partial^{2} c_{0 k}}{\partial X_{j} \partial X_{m}}+\sum_{m=1}^{4} \mathbf{A}_{j m k}^{v} \frac{\partial\left(c_{0 m} c_{0 k}\right)}{\partial X_{j}}\right)-\nabla_{\mathbf{X}}\left\langle\widetilde{p}_{1}\right\rangle_{h},
$$

where we have denoted

$$
\begin{gathered}
\mathbf{D}_{j m k}^{v}=\left\langle\mathbf{V} \times\left(\mathbf{e}_{j} \times \mathbf{G}_{m k}^{v}\right)-\mathbf{H} \times\left(\mathbf{e}_{j} \times \mathbf{G}_{m k}^{h}\right)\right\rangle_{h}, \\
\mathbf{A}_{j m k}^{v}=\left\langle\mathbf{V} \times\left(\mathbf{e}_{j} \times \mathbf{Q}_{m k}^{v}\right)-\mathbf{H} \times\left(\mathbf{e}_{j} \times \mathbf{Q}_{m k}^{h}\right)-\mathbf{S}_{k}^{v} S_{m, j}^{v}+\mathbf{S}_{k}^{h} S_{m, j}^{h}\right\rangle_{h},
\end{gathered}
$$

and from (43),

$$
\frac{\partial\left\langle\mathbf{h}_{0}\right\rangle_{h}}{\partial T}=\eta \nabla_{\mathbf{X}}^{2}\left\langle\mathbf{h}_{0}\right\rangle_{h}-\mathbf{e}_{3} \times \nabla_{\mathbf{X}} \sum_{k=1}^{4}\left(\sum_{m=1}^{2} D_{m k}^{h} \frac{\partial c_{0 k}}{\partial X_{m}}+\sum_{m=1}^{4} A_{m k}^{h} c_{0 m} c_{0 k}\right),
$$

where

$$
\begin{gathered}
D_{m k}^{h}=\left\langle\left(\mathbf{V} \times \mathbf{G}_{m k}^{h}-\mathbf{H} \times \mathbf{G}_{m k}^{v}\right) \cdot \mathbf{e}_{3}\right\rangle, \\
A_{m k}^{h}=\left\langle\left(\mathbf{V} \times \mathbf{Q}_{m k}^{h}-\mathbf{H} \times \mathbf{Q}_{m k}^{v}+\mathbf{S}_{m}^{v} \times \mathbf{S}_{k}^{h}\right) \cdot \mathbf{e}_{3}\right\rangle .
\end{gathered}
$$

The quantities $\mathbf{D}_{j m k}^{v}$ and $D_{m k}^{h}$ describe eddy diffusivity, and $\mathbf{A}_{j m k}^{v}$ and $A_{m k}^{h}$ eddy advection.

Significant simplifications stem from relations (27). The last term in (45) serves to eliminate from this equation the component, parallel to q; consequently, (45) is equivalent to the scalar equation

$$
\lambda \frac{\partial C}{\partial T}=\nu \lambda \frac{\partial^{2} C}{\partial Y^{2}}+\sum_{j=1}^{2} \sum_{k=1}^{4}\left(\sum_{m=1}^{2} \mathbf{q}^{\perp} \cdot \mathbf{D}_{j m k}^{v} q_{j} q_{m} \frac{\partial^{2} c_{0 k}}{\partial Y^{2}}+\sum_{m=1}^{4} \mathbf{q}^{\perp} \cdot \mathbf{A}_{j m k}^{v} q_{j} \frac{\partial\left(c_{0 m} c_{0 k}\right)}{\partial Y}\right) .
$$

All terms in (46) are parallel to $\mathbf{q}^{\perp}$, and, in view of (41), it reduces to the scalar equation

$$
\frac{\partial C}{\partial T}=\mathcal{D}^{h} \frac{\partial^{2} C}{\partial Y^{2}}+\sum_{k=3}^{4} \mathcal{D}_{k}^{h} \frac{\partial^{2} c_{0 k}}{\partial Y^{2}}+\mathcal{A}^{h} \frac{\partial\left(C^{2}\right)}{\partial Y},
$$

where

$$
\begin{gathered}
\mathcal{A}^{h}=\sum_{k=1}^{2} \sum_{m=1}^{2}(-1)^{m+k} A_{m k}^{h} q_{3-m} q_{3-k}, \\
\mathcal{D}^{h}=\eta-\sum_{k=1}^{2} \sum_{m=1}^{2}(-1)^{k} D_{m k}^{h} q_{m} q_{3-k}, \quad \mathcal{D}_{k}^{h}=\sum_{m=1}^{2} D_{m k}^{h} q_{m} .
\end{gathered}
$$

Relations (40) imply the following identities for eddy coefficients in (45)-(46):

$$
D_{14}^{h}=D_{23}^{h}, \quad \mathbf{D}_{j 14}^{v}-\mathbf{D}_{j 23}^{v}=\left\langle-\left(\mathbf{V} \times \mathbf{e}_{3}\right) V_{j}+\left(\mathbf{H} \times \mathbf{e}_{3}\right) H_{j}\right\rangle_{h}
$$

for $j=1,2$. Expressions for coefficients in the last sum in (47) can be simplified by using an identity that follows from (41):

$$
\sum_{j} \mathbf{q}^{\perp} \cdot\left(\mathbf{A}_{j m k}^{v}+\mathbf{A}_{j k m}^{v}\right) q_{j}=\sum_{j} \mathbf{q}^{\perp} \cdot\left\langle\mathbf{S}_{m}^{h} S_{k, j}^{h}-\mathbf{S}_{m}^{v} S_{k, j}^{v}\right\rangle_{h} q_{j},
$$

where $m=3,4$ or $k=3,4$. 
Since the two equations (47) and (48) involve the slow time derivative of the same quantity, $C$, a simpler non-evolutionary equation can be derived, equivalent to any of the two. Multiplying (48) by $\lambda$, subtracting it from (47) and integrating the result in $Y$ we find

$$
\begin{gathered}
\frac{\partial}{\partial Y}\left(\left(\lambda\left(\nu-\mathcal{D}^{h}\right)+\sum_{j=1}^{2} \sum_{m=1}^{2} \sum_{k=1}^{2} \mathbf{q}^{\perp} \cdot \mathbf{D}_{j m k}^{v} q_{j} q_{m}(-1)^{k+1} q_{3-k}\right) C\right. \\
\left.+\sum_{k=3}^{4}\left(-\lambda \mathcal{D}_{k}^{h}+\sum_{j=1}^{2} \sum_{m=1}^{2} \mathbf{q}^{\perp} \cdot \mathbf{D}_{j m k}^{v} q_{j} q_{m}\right) c_{0 k}\right)=\lambda \mathcal{A}^{h} C^{2}-\sum_{j=1}^{2} \sum_{k=1}^{4} \sum_{m=1}^{4} \mathbf{q}^{\perp} \cdot \mathbf{A}_{j m k}^{v} q_{j} c_{0 m} c_{0 k}+K(T),
\end{gathered}
$$

where $K(T)$ is a function of slow time alone. We will consider perturbations (in particular, $c_{0 k}$ ) that are periodic in $Y$; by appropriately rescaling $\varepsilon$, the period then can be made equal to $2 \pi$ (in agreement with (26) ). Averaging (49) in $Y$ over a period uniquely determines $K(T)$.

\subsubsection{The closing evolutionary equation}

Another evolutionary equation will be derived from (42)-(44) owing to the fact that the generalised kernels of the operators $\mathfrak{L}$ and $\mathfrak{L}^{*}$ are six-dimensional. We begin by making a general remark on the means of the perturbation fields, which will be used in the derivations. Let $《 \cdot\rangle$ denote the averaging in $Y$ over a period:

$$
\left\langle\langle f(Y, T)\rangle=\frac{1}{2 \pi} \int_{0}^{2 \pi} f(Y, T) \mathrm{d} Y .\right.
$$

Clearly, application of the two averagings, $\langle\cdot\rangle$ and $\langle\langle\cdot\rangle$, to the flow and magnetic equations obtained at order $\varepsilon^{n+2}$ yields $\partial\left\langle\left\langle\left\langle\mathbf{v}_{n}\right\rangle\right\rangle\right\rangle / \partial T=0$ (there is no contribution from nonlinear terms by virtue of (15)-(18) ) and $\partial\left\langle\left\langle\left\langle\mathbf{h}_{n}\right\rangle\right\rangle\right\rangle / \partial T=0$. Thus, the means over both slow and fast variables of each term of the expansions of the flow and magnetic perturbation are independent of time. We investigate instabilities (if any) of the steady state $\mathbf{W}$ developing in their own right and not due to a mean flow through the layer, or an imposed mean magnetic field, and therefore demand

$$
\left.\left.\left\langle\left\langle\mathbf{v}_{n}\right\rangle\right\rangle\right\rangle=\left\langle\left\langle\mathbf{h}_{n}\right\rangle\right\rangle\right\rangle=0 .
$$

We split $\mathbf{v}_{2}$ and $\mathbf{h}_{2}$, into solenoidal and gradient parts:

$$
\begin{array}{lll}
\mathbf{v}_{2}=\mathbf{v}_{2}^{\text {sol }}+\nabla_{\mathbf{x}} v_{2}^{\mathrm{gr}}, & \nabla_{\mathbf{x}} \cdot \mathbf{v}_{2}^{\text {sol }}=0, & \nabla_{\mathbf{x}}^{2} v_{2}^{\mathrm{gr}}=-\nabla_{\mathbf{x}} \cdot \mathbf{v}_{1}, \\
\mathbf{h}_{2}=\mathbf{h}_{2}^{\text {sol }}+\nabla_{\mathbf{x}} h_{2}^{\mathrm{gr}}, & \nabla_{\mathbf{x}} \cdot \mathbf{h}_{2}^{\text {sol }}=0, & \nabla_{\mathbf{x}}^{2} h_{2}^{\mathrm{gr}}=-\nabla_{\mathbf{x}} \cdot \mathbf{h}_{1},
\end{array}
$$

where we have used relations (17) and (18) for $n=2$. Upon substituting these sums into (42)-(44), we recast this system as

$$
\mathfrak{L w}_{2}^{\text {sol }}=\partial \mathbf{w}_{0} / \partial T-\mathbf{F},
$$

where $\mathbf{w}_{2}^{\text {sol }}=\left(\mathbf{v}_{2}^{\text {sol }}, \mathbf{h}_{2}^{\text {sol }}, \theta_{2}\right)$ and $\mathbf{F}$ denotes the sum of all the remaining terms in these equations. Scalar multiplying (53) by $\mathbf{S}_{j}^{*}, j=1,2$, we find using (23):

$$
\frac{\partial}{\partial T}\left\langle\mathbf{w}_{0} \cdot \mathbf{S}_{j}^{*}\right\rangle-\left\langle\mathbf{F} \cdot \mathbf{S}_{j}^{*}\right\rangle=\left\langle\mathfrak{L} \mathbf{w}_{2}^{\text {sol }} \cdot \mathbf{S}_{j}^{*}\right\rangle=\left\langle\mathbf{w}_{2}^{\text {sol }} \cdot \mathfrak{L}^{*} \mathbf{S}_{j}^{*}\right\rangle=\sum_{m=1}^{2} u_{m j}^{\prime}\left\langle v_{2, m}\right\rangle+\left\langle h_{2, j}\right\rangle
$$

(note that, by our constructions in section 2.2.1, relation (20) defining the adjoint operator $\mathfrak{L}^{*}$ is only valid for fields $\mathbf{w}$ and $\mathbf{w}^{*}$, whose flow and magnetic field components are solenoidal in the fast spatial variables - this has forced us to perform the Helmholtz decompositions (51) -(52)). Now, by virtue of (24),

$$
\sum_{j=1}^{2}\left(\frac{\partial}{\partial T}\left\langle\mathbf{w}_{0} \cdot \mathbf{S}_{j}^{*}\right\rangle-\left\langle\mathbf{F} \cdot \mathbf{S}_{j}^{*}\right\rangle\right) \mathbf{e}_{j}=-\mathcal{U}^{-1}\left\langle\mathbf{v}_{2}\right\rangle_{h}+\left\langle\mathbf{h}_{2}\right\rangle_{h}
$$


However, by (15) and (16) for $j=2,\left\langle\mathbf{v}_{2}\right\rangle_{h}$ and $\left\langle\mathbf{h}_{2}\right\rangle_{h}$ are solenoidal in the slow variable $\mathbf{X}$, and, since their spatial dependence is exclusively on $Y=\mathbf{X} \cdot \mathbf{q}$, they are parallel to $\mathbf{q}^{\perp}$ (Fourier expansion in $Y$ can be used to show this for zero-mean fields; see (50) for $n=2$ ). Furthermore, since $\mathbf{q}^{\perp}$ is an eigenvector of $\mathcal{U}$, the r.h.s. of (54) is parallel to $\mathbf{q}^{\perp}$. Thus, scalar multiplying (54) by $\mathbf{q}$, we obtain a closed equation in $c_{0 k}$ :

$$
\sum_{j=1}^{2}\left(\sum_{k=1}^{4} \frac{\partial c_{0 k}}{\partial T}\left\langle\mathbf{S}_{k} \cdot \mathbf{S}_{j}^{*}\right\rangle-\left\langle\mathbf{F} \cdot \mathbf{S}_{j}^{*}\right\rangle\right) q_{j}=0
$$

It can be simplified using relations (22) and (23):

$$
\left\langle\mathbf{S}_{k+2} \cdot \mathbf{S}_{j}^{*}\right\rangle=-\left\langle\mathfrak{L}\left(\mathbf{e}_{k}, 0,0\right) \cdot \mathbf{S}_{j}^{*}\right\rangle=-u_{k j}^{\prime} .
$$

Since $\mathbf{q}$ is an eigenvector of the matrix (24),

$$
\sum_{j=1}^{2}\left\langle\mathbf{S}_{k+2} \cdot \mathbf{S}_{j}^{*}\right\rangle q_{j}=-\sum_{j=1}^{2} u_{k j}^{\prime} q_{j}=\mathbf{e}_{k} \cdot\left(\mathcal{U}^{t}\right)^{-1} \mathbf{q}=\frac{q_{k}}{\lambda^{\prime}}
$$

By virtue of (27), (55) takes the form

$$
\frac{\partial}{\partial T}\left(\sum_{j=1}^{2} q_{j}\left\langle\left(q_{2} \mathbf{S}_{1}-q_{1} \mathbf{S}_{2}\right) \cdot \mathbf{S}_{j}^{*}\right\rangle C+\frac{1}{\lambda^{\prime}} \sum_{k=3}^{4} q_{k-2} c_{0 k}\right)=\sum_{j=1}^{2} q_{j}\left\langle\mathbf{F} \cdot \mathbf{S}_{j}^{*}\right\rangle .
$$

It is straightforward to calculate the scalar products in the r.h.s. of this equation, using the expressions for the two leading terms in the expansion of perturbation, that were found in sections 2.2.1 and 2.3. The result has a simple structure, but involves bulky coefficients:

$$
\begin{aligned}
\left\langle\mathbf{F} \cdot \mathbf{S}_{j}^{*}\right\rangle=\sum_{n=1}^{2} \sum_{m=1}^{2} & \sum_{k=1}^{4} \frac{\partial^{2} c_{0 k}}{\partial X_{n} \partial X_{m}} f_{n m k j}^{1}+\sum_{n=1}^{2} \sum_{m=1}^{4} \sum_{k=1}^{4} \frac{\partial c_{0 k}}{\partial X_{n}} c_{0 m} f_{n m k j}^{2} \\
& +\sum_{n=1}^{4} \sum_{m=1}^{4} \sum_{k=1}^{4} c_{0 n} c_{0 m} c_{0 k} f_{n m k j}^{3}
\end{aligned}
$$

where

$$
\begin{aligned}
f_{n m k j}^{1}= & \left\langle\left(2 \nu \partial \mathbf{G}_{m k}^{v} / \partial x_{n}+\delta_{m}^{n} \nu \mathbf{S}_{k}^{v}+\mathbf{V} \times\left(\mathbf{e}_{n} \times \mathbf{G}_{m k}^{v}\right)-\mathbf{H} \times\left(\mathbf{e}_{n} \times \mathbf{G}_{m k}^{h}\right)\right) \cdot \mathbf{S}_{j}^{* v}\right. \\
& -G_{m k}^{p} S_{j, n}^{* v}+\left(2 \eta \partial \mathbf{G}_{m k}^{h} / \partial x_{n}+\delta_{m}^{n} \eta \mathbf{S}_{k}^{h}+\mathbf{e}_{n} \times\left(\mathbf{V} \times \mathbf{G}_{m k}^{h}-\mathbf{H} \times \mathbf{G}_{m k}^{v}\right)\right) \cdot \mathbf{S}_{j}^{* h} \\
& \left.+\left(2 \kappa \partial G_{m k}^{\theta} / \partial x_{n}+\delta_{m}^{n} \kappa S_{k}^{\theta}-V_{n} G_{m k}^{\theta}\right) S_{j}^{* \theta}-\mathfrak{L}\left(\nabla \widehat{G}_{m k n}^{v}, \nabla \widehat{G}_{m k n}^{h}, 0\right) \cdot \mathbf{S}_{j}^{*}\right\rangle, \\
f_{n m k j}^{2}= & \left\langle\left( 4 \nu \partial \mathbf{Q}_{m k}^{v} / \partial x_{n}+2 \mathbf{V} \times\left(\mathbf{e}_{n} \times \mathbf{Q}_{m k}^{v}\right)-2 \mathbf{H} \times\left(\mathbf{e}_{n} \times \mathbf{Q}_{m k}^{h}\right)\right.\right. \\
& +\mathbf{S}_{m}^{v} \times\left(\nabla_{\mathbf{x}} \times \mathbf{G}_{n k}^{v}+\mathbf{e}_{n} \times \mathbf{S}_{k}^{v}\right)-\mathbf{S}_{m}^{h} \times\left(\nabla_{\mathbf{x}} \times \mathbf{G}_{n k}^{h}+\mathbf{e}_{n} \times \mathbf{S}_{k}^{h}\right) \\
& \left.+\mathbf{G}_{n k}^{v} \times\left(\nabla_{\mathbf{x}} \times \mathbf{S}_{m}^{v}\right)-\mathbf{G}_{n k}^{h} \times\left(\nabla_{\mathbf{x}} \times \mathbf{S}_{m}^{h}\right)\right) \cdot \mathbf{S}_{j}^{* v}-2 Q_{m k}^{p} S_{j, n}^{* v} \\
& +\left(4 \eta \partial \mathbf{Q}_{m k}^{h} / \partial x_{n}+\mathbf{e}_{n} \times\left(2 \mathbf{V} \times \mathbf{Q}_{m k}^{h}-2 \mathbf{H} \times \mathbf{Q}_{m k}^{v}+\mathbf{S}_{m}^{v} \times \mathbf{S}_{k}^{h}+\mathbf{S}_{k}^{v} \times \mathbf{S}_{m}^{h}\right)\right. \\
& \left.+\nabla_{\mathbf{x}} \times\left(\mathbf{S}_{m}^{v} \times \mathbf{G}_{n k}^{h}-\mathbf{S}_{m}^{h} \times \mathbf{G}_{n k}^{v}\right)\right) \cdot \mathbf{S}_{j}^{* h}+\left(4 \kappa \partial Q_{m k}^{\theta} / \partial x_{n}-2 V_{n} Q_{m k}^{\theta}\right. \\
& \left.\left.-\left(\mathbf{G}_{n k}^{v} \cdot \nabla_{\mathbf{x}}\right) S_{m}^{\theta}-\left(\mathbf{S}_{m}^{v} \cdot \nabla_{\mathbf{x}}\right) G_{n k}^{\theta}-S_{m, n}^{v} S_{k}^{\theta}\right) S_{j}^{* \theta}-\mathfrak{L}\left(\nabla \widehat{Q}_{m k n}^{v}, \nabla \widehat{Q}_{m k n}^{h}, 0\right) \cdot \mathbf{S}_{j}^{*}\right\rangle,
\end{aligned}
$$




$$
\begin{aligned}
f_{n m k j}^{3}= & \left\langle\left(\mathbf{S}_{n}^{v} \times\left(\nabla_{\mathbf{x}} \times \mathbf{Q}_{m k}^{v}\right)-\mathbf{S}_{n}^{h} \times\left(\nabla_{\mathbf{x}} \times \mathbf{Q}_{m k}^{h}\right)+\mathbf{Q}_{m k}^{v} \times\left(\nabla_{\mathbf{x}} \times \mathbf{S}_{n}^{v}\right)\right.\right. \\
& \left.-\mathbf{Q}_{m k}^{h} \times\left(\nabla_{\mathbf{x}} \times \mathbf{S}_{n}^{h}\right)\right) \cdot \mathbf{S}_{j}^{* v}+\left(\nabla_{\mathbf{x}} \times\left(\mathbf{S}_{n}^{v} \times \mathbf{Q}_{m k}^{h}-\mathbf{S}_{n}^{h} \times \mathbf{Q}_{m k}^{v}\right)\right) \cdot \mathbf{S}_{j}^{* h} \\
& \left.-\left(\left(\mathbf{Q}_{m k}^{v} \cdot \nabla_{\mathbf{x}}\right) S_{n}^{\theta}+\left(\mathbf{S}_{n}^{v} \cdot \nabla_{\mathbf{x}}\right) Q_{m k}^{\theta}\right) S_{j}^{* \theta}\right\rangle,
\end{aligned}
$$

$\delta_{m}^{n}$ is the Kronecker symbol, and $\widehat{G}_{m k n}^{v}, \widehat{G}_{m k n}^{h}, \widehat{Q}_{m k n}^{v}$ and $\widehat{Q}_{m k n}^{h}$ are space-periodic solutions to the Poisson equations

$$
\nabla^{2} \widehat{G}_{m k n}^{v}=G_{m k, n}^{v}, \quad \nabla^{2} \widehat{G}_{m k n}^{h}=G_{m k, n}^{h}, \quad \nabla^{2} \widehat{Q}_{m k n}^{v}=Q_{m k, n}^{v}, \quad \nabla^{2} \widehat{Q}_{m k n}^{h}=Q_{m k, n}^{h} .
$$

The following identities for the coefficients $f$ prove useful:

1. For $n=3$ or 4 , and any $m, k$ and $j$, straightforward algebra with application of (21) and (41) yields two relations:

$$
f_{n m k j}^{3}+f_{n k m j}^{3}=2\left\langle\left(\partial \mathfrak{L} \mathbf{Q}_{m k} / \partial x_{n-2}-\mathfrak{L}\left(\partial \mathbf{Q}_{m k} / \partial x_{n-2}\right)\right) \cdot \mathbf{S}_{j}^{*}\right\rangle
$$

and

$$
f_{m n k j}^{3}+f_{m k n j}^{3}+f_{k m n j}^{3}+f_{k n m j}^{3}=-2\left\langle\left(\partial \mathfrak{L} \mathbf{Q}_{m k} / \partial x_{n-2}\right) \cdot \mathbf{S}_{j}^{*}\right\rangle .
$$

Consequently, no terms involving factors $c_{03}$ or $c_{04}$ are present in the last sum in the r.h.s. of (58).

2. For any $n, j=1,2$,

$$
f_{n 34 j}^{2}=f_{n 43 j}^{2}
$$

since by inspection

$$
f_{n 34 j}^{2}-f_{n 43 j}^{2}=\left\langle\mathfrak{L}\left(\frac{\partial \mathbf{G}_{n 3}}{\partial x_{2}}-\frac{\partial \mathbf{G}_{n 4}}{\partial x_{1}}\right) \cdot \mathbf{S}_{j}^{*}\right\rangle=0 .
$$

Derivation of equations for amplitudes is completed by performing the following operations: $(i)$ substitute in the r.h.s. of (49) and (58) the variables $c_{01}$ and $c_{02}$, using the second relation in (27); (ii) in the l.h.s. of (57), eliminate the term, proportional to $\partial C / \partial T$, by subtracting the appropriate multiple of (48); ( modified (57) is proportional to $\partial \xi_{2} / \partial T$ ), and $\xi_{3}$ denoting the linear combination of $C, c_{03}$ and $c_{04}$, whose derivative in $Y$ is the l.h.s. of (49); $(i v)$ in the modified (157), replace $\partial^{2} \xi_{3} / \partial Y^{2}$ by a derivative of a quadratic form of $\xi_{i}$ applying the non-evolutionary equation (49). Upon expressing the r.h.s. of (48), the modified (57) and (49) in terms of $\xi_{i}$, we obtain with the use of (59) three equations,

$$
\begin{aligned}
& \frac{\partial \xi_{1}}{\partial T}=\frac{\partial^{2}}{\partial Y^{2}} \sum_{k=1}^{3} \beta_{1 k} \xi_{k}+\gamma_{1} \frac{\partial \xi_{1}^{2}}{\partial Y}, \\
& \frac{\partial \xi_{2}}{\partial T}=\frac{\partial^{2}}{\partial Y^{2}}\left(\beta_{21} \xi_{1}+\beta_{22} \xi_{2}\right)+\sum_{i, j} \gamma_{2 i j} \xi_{i} \frac{\partial \xi_{j}}{\partial Y}+\sigma \xi_{1}^{3}, \\
& \frac{\partial \xi_{3}}{\partial Y}=\sum_{i, j} \gamma_{3 i j} \xi_{i} \xi_{j}-\left\langle\left\langle\sum_{i, j} \gamma_{3 i j} \xi_{i} \xi_{j}\right\rangle\right\rangle .
\end{aligned}
$$

The coefficients $\beta, \gamma$ and $\sigma$ can be calculated following transformations $(i)-(i v)$ and using the expressions that we have obtained for the coefficients of equations (48), (57) and (49) in terms of solutions to the three auxiliary problems and eigenmodes from the generalised kernel of $\mathfrak{L}^{*}$, the adjoint to the operator of linearisation.

We find from (60) that $\left\langle\left\langle\xi_{1}\right\rangle\right\rangle$ does not depend on the slow time; hence, in view of (150) for $n=0$ and the first equation in (27), $\left\langle\left\langle\xi_{1}\right\rangle=0\right.$. Relation (62) does not completely specify $\xi_{3}$, since it reduces to a trivial identity for the zero wave-number Fourier harmonic (in $Y$ ) of (62). We thus lack an equation to close the system (60) $-(\underline{62})$. 


\subsubsection{The closing evolutionary equation}

To obtain the closing relation for the leading-order terms of the perturbation, we have to consider the equations obtained at even higher orders. Such a situation is often encountered in the dynamo theory, for instance, one has to descend by 2 orders of magnitude to derive the magnetic $\alpha$-effect in the classical almost-axisymmetric Braginsky dynamo [3].

In order to derive the missing equation, we average (154) in $Y$, apply condition (150) for $n=2$, and find

$$
\left.\sum_{k=3}^{4} \frac{\partial\left\langle\left\langle c_{0 k}\right\rangle\right\rangle}{\partial T}\left\langle\mathbf{S}_{k} \cdot \mathbf{S}_{j}^{*}\right\rangle=\langle\langle\mathbf{F}\rangle\rangle \cdot \mathbf{S}_{j}^{*}\right\rangle
$$

The component of (54), parallel to $\mathbf{q}$, has been fully explored in Section 2.4.1; we consider now the component, perpendicular to $\mathbf{q}$. Scalar multiplication of (63) by $\mathbf{q}^{\perp}$ and the use of (566) yields

$$
\sum_{j=1}^{2}\left(\sum_{k=1}^{2} u_{k j}^{\prime} \frac{\partial}{\partial T}\left\langle\left\langle c_{0, k+2}\right\rangle\right\rangle+\left\langle\langle\mathbf{F}\rangle \cdot \mathbf{S}_{j}^{*}\right\rangle\right)(-1)^{j} q_{3-j}=0
$$

We express here the mean of (158),

$$
\left\langle\langle\mathbf{F}\rangle \cdot \mathbf{S}_{j}^{*}\right\rangle=\sum_{n=1}^{2} \sum_{m=1}^{4} \sum_{k=1}^{4}\left\langle\left\langle\frac{\partial c_{0 k}}{\partial X_{n}} c_{0 m}\right\rangle\right\rangle f_{n m k j}^{2}+\sum_{n=1}^{2} \sum_{m=1}^{2} \sum_{k=1}^{2}\left\langle\left\langle c_{0 n} c_{0 m} c_{0 k}\right\rangle\right\rangle f_{n m k j}^{3},
$$

in terms of $\xi_{i}$ using (59):

$$
\frac{\partial}{\partial T}\left\langle\left\langle\mu_{1}^{\prime} \xi_{2}+\mu_{2}^{\prime} \xi_{3}\right\rangle\right\rangle=\left\langle\left\langle\xi_{1} \frac{\partial}{\partial Y}\left(\gamma_{2}^{\prime} \xi_{2}+\gamma_{3}^{\prime} \xi_{3}\right)\right\rangle\right\rangle+\sigma^{\prime}\left\langle\left\langle\xi_{1}^{3}\right\rangle\right\rangle
$$

and subtract from this equation (61) averaged in $Y$ and multiplied by $\sigma^{\prime} / \sigma$. This finally yields the closing equation:

$$
\frac{\partial}{\partial T}\left\langle\mu_{1} \xi_{2}+\mu_{2} \xi_{3}\right\rangle=\left\langle\left\langle\xi_{1} \frac{\partial}{\partial Y}\left(\gamma_{42} \xi_{2}+\gamma_{43} \xi_{3}\right)\right\rangle\right\rangle .
$$

Equations (60)- (62) and (65) comprise a closed system in $\xi_{i}$, equivalent to the system of amplitude equations.

\section{Numerical results}

In this section, we present results of numerical investigation of the two-scale weakly nonlinear stability of space-periodic steady-state convective dynamos in a horizontal layer of rotating conducting fluid, that comprise the branch labelled $S_{8}^{R 1}$ in [7]. The branch is parameterised by the Taylor number, Ta, and exists in the interval $673.7 \leq \mathrm{Ta} \leq 704$. These convective MHD steady states are symmetric about the vertical axis, $x_{3}$, and hence the significant (acting on the amplitudes) $\alpha$-effect is absent. Dynamos from this branch have the group of symmetries $\mathbf{D}_{2}$, the second group generator being the composition of reflection about the midplane and the shift by half a period in the horizontal direction $x_{1}$. For this group of symmetries, the matrix $\mathcal{U}$ is non-zero, this invalidating the multiscale analysis [33] of large-scale perturbations of these dynamos.

We have refined the steady states from the branch $\mathrm{S}_{8}^{\mathrm{R} 1}$ with the resolution of $64^{2} \times 32$ Fourier harmonics. When solving the auxiliary problems and computing neutral eigenmodes of $\mathfrak{L}^{*}$ (the adjoint to the operator of linearisation), solutions in the form of truncated Fourier series have been sought. Pseudospectral methods [12] have been used (resolution: $96^{2} \times 48$ Fourier harmonics before dealiasing) for computing various products encountered in the operator $\mathfrak{L}$. The code [10] realising the biconjugate gradients stabilised method $\operatorname{BiCGstab}(\ell)$ [23 25] for $\ell=2$ has been applied to solve the linear system of equations for the Fourier coefficients. Table 1 shows the values of the coefficients in the system of amplitude equations for $\mathrm{Ta}=675$. 


\subsection{Linear stability of constant amplitudes}

The behaviour of solutions depends crucially on whether the evolutionary equations (600)-(61) are parabolic. They constitute a parabolic (sub)system, when real parts of both eigenvalues of the matrix

$$
\left[\begin{array}{ll}
\beta_{11} & \beta_{12} \\
\beta_{21} & \beta_{22}
\end{array}\right]
$$

are positive. Otherwise, the second-order partial differential operator in the r.h.s. of (60)- (61) would cause a superexponential growth of solutions in time (unless it is suppressed by nonlinear terms - but they are more likely to boost rather than inhibit this growth). The term $\beta_{13} \partial^{2} \xi_{3} / \partial Y^{2}$ is not involved in this analysis, because (62) is a non-evolutionary equation. The eigenvalues $d_{i}$ shown in Table 2 reveal a high variability of the parameter values controlling the perturbation despite the interval, in Ta, of existence of the branch is short. At the left end of this interval for $\mathrm{S}_{8}^{\mathrm{R} 1}, d_{1}>0$ but $d_{2}<0$, indicating a rapid (in the slow time) instability. In the remaining part of the interval, both $d_{i}>0$ and hence the second-order operator acts as the stabilising diffusion.

Clearly, $\xi_{1}=0$ and any constant in time and space $\xi_{2}$ and $\xi_{3}$ (or, equivalently, $C=0$ and

Table 1: Coefficients in the amplitude equations for $\mathrm{Ta}=675$.

\begin{tabular}{|c|c|lll|}
\hline Eq. & Coeff. & Values & \\
\hline ([60) & $\beta_{1 k}$ & 0.884249123107103, & 0.973164220817207, & 0.002542972607168343 \\
& $\gamma_{1}$ & 0.588162048986537 & \\
\hline ([61) & $\beta_{2 k}$ & 0.366161979311609, & 4.15859432509866 & \\
& $\gamma_{2 i j}$ & 0.806971618405987, & -4.23666924855939, & 0.158691136470146, \\
& & -4.82055714849942, & -42.2469280336739, & 0.135854143000776, \\
& & 0.209741278940542, & 0.135854143000747, & 0.003874598372548109, \\
& $\sigma$ & 0.192034252840662 & \\
\hline (62) & $\gamma_{3 i j}$ & -7.08024457707350, & 8.63396618933175, & -0.500706409449314, \\
& & 8.63396618933175, & 85.1114936402045, & 0.385556218828258, \\
& & -0.500706409449314, & 0.385556218828260, & -0.03382831817117658 \\
\hline (65) & $\mu_{i}$ & $1,-0.002659134450504667$ & \\
& $\gamma_{4 i}$ & 0.596884895067222, & -0.04846657396378542 \\
\hline
\end{tabular}

Table 2: Eigenvalues of matrix ([66), $d_{i}$, for two unit vectors $\mathbf{q}$ (such that $\mathbf{q}^{\perp}$ are eigenvectors of matrix $\mathcal{U}$ ).

\begin{tabular}{|c|c|c|c|}
\hline $\mathrm{Ta}$ & $q$ & $d_{1}$ & $d_{2}$ \\
\hline \multirow[t]{2}{*}{673.7} & $\mathbf{q}^{1}=(0.998515,0.054469)$ & 0.763328 & 4.298197 \\
\hline & $\mathbf{q}^{2}=(0.822115,0.569320)$ & 0.264885 & -261.047947 \\
\hline \multirow[t]{2}{*}{675} & $\mathbf{q}^{1}=(0.998613,0.052645)$ & 0.778817 & 4.264026 \\
\hline & $\mathbf{q}^{2}=(0.825155,0.564905)$ & 0.270588 & -361.244316 \\
\hline \multirow[t]{2}{*}{680} & $\mathbf{q}^{1}=(0.998954,0.045718)$ & 0.889724 & 4.175111 \\
\hline & $\mathbf{q}^{2}=(0.836586,0.547835)$ & 0.289251 & 1326.420209 \\
\hline \multirow[t]{2}{*}{690} & $\mathbf{q}^{1}=(0.999477,0.032314)$ & 1.588269 & 4.598703 \\
\hline & $\mathbf{q}^{2}=(0.858485,0.512837)$ & 0.297058 & 201.124913 \\
\hline \multirow[t]{2}{*}{700} & $\mathbf{q}^{1}=(0.999808,0.019574)$ & 2.518614 & 16.785099 \\
\hline & $\mathbf{q}^{2}=(0.879679,0.475566)$ & 0.174285 & 212.326992 \\
\hline \multirow[t]{2}{*}{703} & $\mathbf{q}^{1}=(0.999873,0.015897)$ & 2.597766 & 65.565570 \\
\hline & $\mathbf{q}^{2}=(0.886016,0.463653)$ & 0.057593 & 281.662699 \\
\hline
\end{tabular}




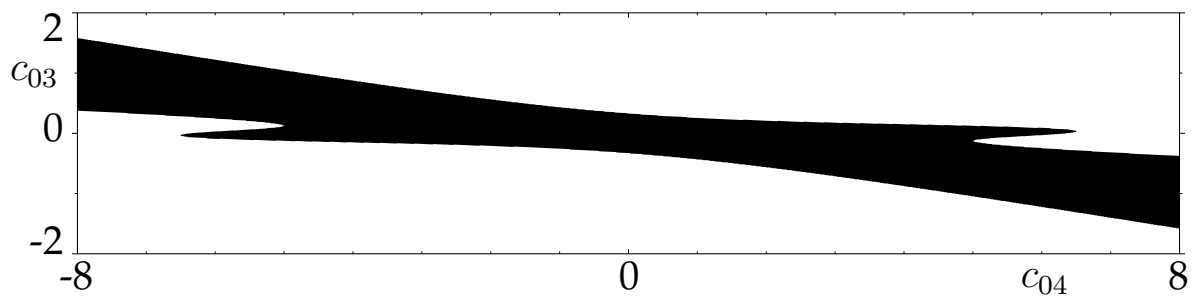

Figure 1: The region of linear stability (black) of constant solutions to the system of amplitude equations in the rectangle $\left|c_{03}\right| \leq 2,\left|c_{04}\right| \leq 8, C=0$.

any constant $c_{03}$ and $c_{04}$ ) are solutions to the system of amplitude equations (60) -(62), (65). In other words, any linear combination of the neutral modes $\mathbf{S}_{k+2}$ (21) with constant coefficients is a solution to the stability problem in the broader class of weakly nonlinear large-scale perturbations. The condition of parabolicity of the linear operator in (60)-(61) is the condition of stability of the solution $\xi_{i}=0$ (i.e., $\left.C=c_{03}=c_{04}=0\right)$. A constant solution $\left(0, \xi_{2}, \xi_{3}\right)$ is linearly stable if $\operatorname{Re} \varrho<0$ for both roots $\varrho$ of the quadratic equation

$$
\operatorname{det}\left[\begin{array}{ccc}
\beta_{11}+\varrho & \beta_{12} & \beta_{13} \\
1 \beta_{21}+\Gamma_{21} & 1 \beta_{22}+\Gamma_{22}+1 \varrho & \Gamma_{23} \\
\Gamma_{31} & \Gamma_{32} & -1
\end{array}\right]=0
$$

(see Fig. 1). Here we have denoted

$$
\Gamma_{2 k}=\sum_{j=2}^{3} \gamma_{2 j k} \xi_{j}, \quad \Gamma_{3 k}=\frac{\left(\sum_{j=2}^{3}\left(\gamma_{3 j k}+\gamma_{3 k j}\right) \xi_{j}\right)\left(\sum_{j=2}^{3}\left(\gamma_{3 j 3}+\gamma_{33 j}\right) \xi_{j}\right)-1 \sum_{j=2}^{3}\left(\gamma_{3 j k}+\gamma_{3 k j}\right) \xi_{j}}{1+\left(\sum_{j=2}^{3}\left(\gamma_{3 j 3}+\gamma_{33 j}\right) \xi_{j}\right)^{2}} .
$$

\subsection{Two types of behaviour of amplitudes}

We report now results of numerical investigation of the system of amplitude equations (60)(62), (65). Solutions, 2 $\pi$-periodic in $Y$, have been sought as truncated Fourier series in the spatial variable. Pseudospectral methods [12] have been applied. The presence of the cubic term in (61) necessitates performing the dealiasing by discarding a half of the highest-wave-number harmonics, rather than $1 / 3$ of them as stipulated by the standard $3 / 2$ rule [17] (when evaluating quadratic terms). The second-order implicit midpoint method has been used to integrate the system in time. In terms of the Fourier coefficients of the variables $\xi_{s}(Y, T)$ denoted by $\widehat{\boldsymbol{\xi}}(T)=\left(\widehat{\xi}_{1}(T), \ldots, \widehat{\xi}_{3 M}(T)\right)$, the system takes the form

$$
\begin{aligned}
\frac{\partial \widehat{\xi}_{m}}{\partial T} & =f_{m}(\widehat{\boldsymbol{\xi}}), & & 1 \leq m \leq 2 M+1, \\
0 & =f_{m}(\widehat{\boldsymbol{\xi}}), & & 2 M+2 \leq m \leq 3 M
\end{aligned}
$$

(here evolutionary equations (67) stem from (60), (61) and (65), and (68) from (62)). In this notation, the midpoint method propagates solution $\widehat{\boldsymbol{\xi}}^{(n)}$ at time $t_{n}$ a step forward to $\widehat{\boldsymbol{\xi}}^{(n+1)}$ at time $t_{n+1}=t_{n}+d t$ according to the equations

$$
\begin{aligned}
\widehat{\xi}_{m}^{(n+1)}-\widehat{\xi}_{m}^{(n)}-d t f_{m}\left(\frac{\widehat{\boldsymbol{\xi}}^{(n+1)}+\widehat{\boldsymbol{\xi}}^{(n)}}{2}\right)=0, & 1 \leq m \leq 2 M+1, \\
f_{m}\left(\widehat{\boldsymbol{\xi}}^{(n+1)}\right)=0, & 2 M+2 \leq m \leq 3 M .
\end{aligned}
$$


To solve (69)-(70), we have used the routine newt [21] that realises a quasi-Newton method (which is a combination of the Newton method with a minimisation technique). The initial conditions are required to satisfy (70). Mostly, a time step $d t=10^{-4}$ was used. A number of runs have been also performed using the standard fourth-order Runge-Kutta method and the time step $d t=10^{-3}$, with (70) being solved at each substep. Comparison of the results obtained by the two methods has shown a satisfactory quantitative agreement.

Solutions to amplitude equations (60) -(62) and (65) apparently do not obey any conservation law and thus, in principle, can saturate at constant values or grow to infinity. In fact, we have not found any other patterns of behaviour.

Let us now discuss in detail a run for $\mathrm{Ta}=675$ and $\mathbf{q}=\mathbf{q}^{1}$ (see Table21), which we will refer to as "the main sample run". In a preparation of a blow-up, maxima of all the three amplitudes increase in time indefinitely; a graph of an amplitude near its maximum turns into a spike, travelling along the $Y$-axis at an increasing speed and finally growing to very large values at a finite time (see a typical behaviour in Fig. 2 and a video stored at arXiv.org as an ancillary file Ta675.mp4). Simulated solutions were truncated to 256 Fourier harmonics in the spatial variable (i.e., they involved wave numbers up to 127, and computation of nonlinear terms involved 512 harmonics before dealiasing). The noise in the graphs of absolute values of the Fourier coefficients of the amplitudes (panels (j)-(l) in Fig. 2) at $T=31$ for wave numbers exceeding 95 indicates, that such a resolution is excessive when computing with the double precision up to this time. In the course of evolution, the energy gets eventually almost evenly distributed between all Fourier harmonics (panels (j)-(l) in Fig. 2); at the final stage, amplitude $C$ resembles a periodically-replicated $\delta$-function moving at a constant speed. (Of course, at this stage the numerical solution is underresolved and cannot be fully trusted any more.) A common feature of nonlinear partial differential equations of the first order such as the inviscid Burgers equation is development of a jump due to intersection of characteristics; in the present case the singularity can be rather interpreted as a derivative of a function, experiencing a jump. However, addition of a small diffusivity into the Burgers equation smooths out the jumps, which is not the case in the present system of amplitude equations, where diffusion is present and is not infinitesimal.

Blow-ups are well-known to be linked with self-similar solutions (see, e.g., [2, 9]). It is easy to check that equations (60)-(62) and (65) admit self-similar solutions of the form

$$
\left(C, c_{03}, c_{04}\right)=\left(T_{\star}-T\right)^{-1 / 2} \Phi\left(Y\left(T_{\star}-T\right)^{-1 / 2}\right)
$$

(see [20]). To examine, whether the solution has this asymptotics near the singularity, we plot in Fig. 3 graphs of the quantities

$$
M_{f, \max }(T)=1 / \max _{Y} f(Y, T), \quad M_{f, \min }(T)=1 / \min _{Y} f(Y, T),
$$

as well $M_{f, \max }^{2}(T)$ and $M_{f, \text { min }}^{2}(T)$; here the function $f$ is any amplitude $C, c_{03}$ and $c_{04}$. (The maxima and their positions are determined applying the quadratic interpolation.) The graphs reveal that the factor $\left(T_{\star}-T\right)^{-1 / 2}$ may indeed correctly describe the growth of the amplitudes at intermediate times preceding the blow-up $(31 \leq T \leq 33$ for the maxima of the solution under consideration, as seen in panels (g)-(i) of Fig. 3). (This is not seen as clearly in panels (j)-(l) of Fig. 3 for the minima, because $\min _{Y} c_{04}(Y, T)$ vanishes at $T \approx 33.66$, forcing us to plot $M_{f, \min }(T)=1 / \min _{Y} f(Y, T)$ for a shorter time interval.) However, further on, at times immediately preceding the blow-up, the maxima and minima behave as $\left(T_{\star}-T\right)^{-1}$ (as follows from graphs in panels (a)-(f) of Fig. 31). The graphs in panels (a)-(c) for the maxima of amplitudes and in panels (d)-(f) for their minima coherently indicate that the blow-up occurs at $T_{\star} \approx 34.17$.

To see how well the self-similar solution (71) approximates near the singularity the spatial dependence of the amplitudes, we have explored the behaviour of $P_{f}(T)$, the position of the maximum 

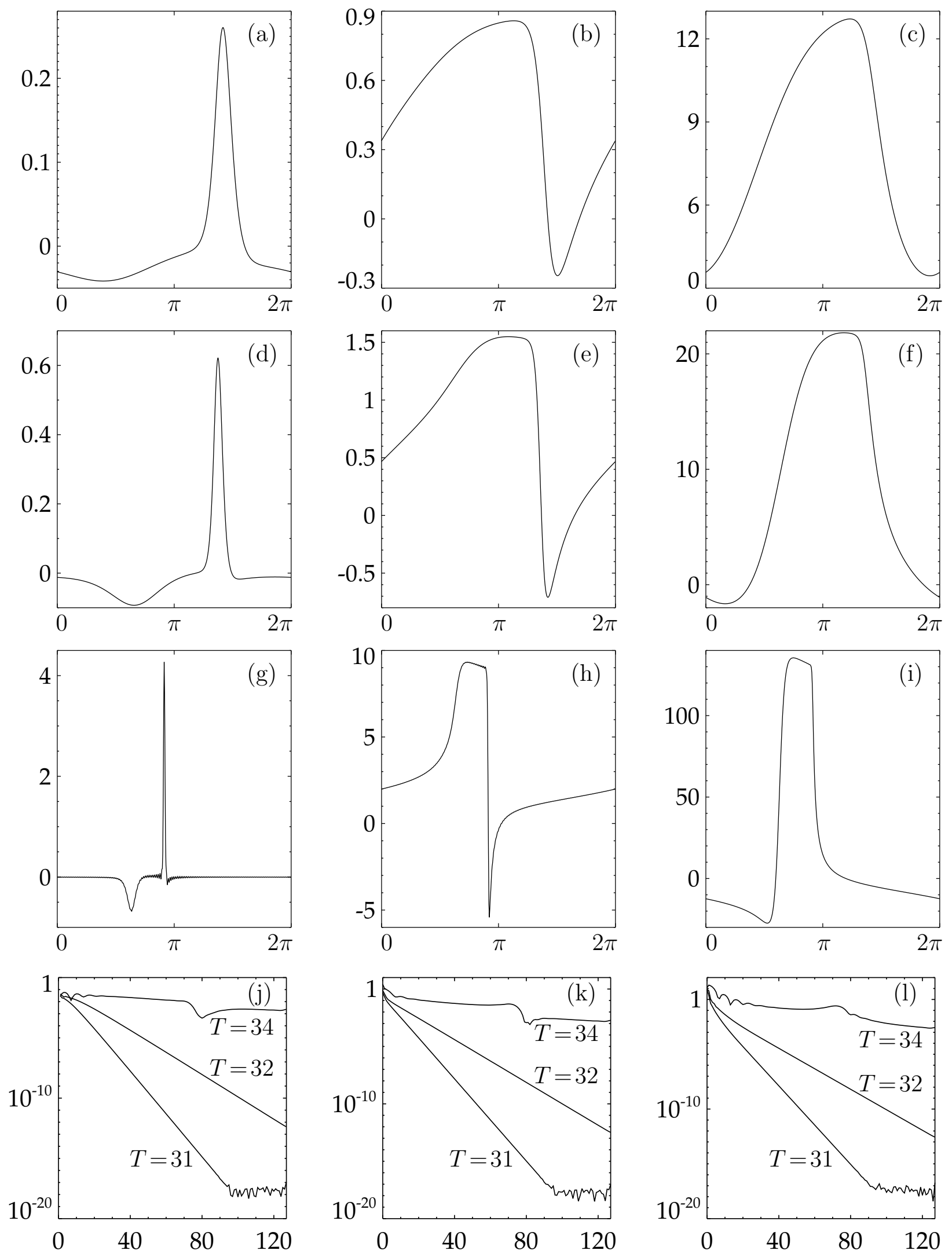

Figure 2: The amplitudes (vertical axis) $C$ (panels (a), (d), (g)), $c_{03}((\mathrm{~b}),(\mathrm{e}),(\mathrm{h}))$ and $c_{04}$ ((c), (f), (i)) at $T=31$ $((\mathrm{a})-(\mathrm{c})), T=33((\mathrm{~d})-(\mathrm{f}))$ and $T=34((\mathrm{~g})-(\mathrm{i}))$; horizontal axis: $Y$. Absolute values of the Fourier coefficients of the amplitudes (vertical axis) $C(\mathrm{j}), c_{03}(\mathrm{k})$ and $c_{04}(\mathrm{l})$ at the same times $T$; horizontal axis: wave number. 

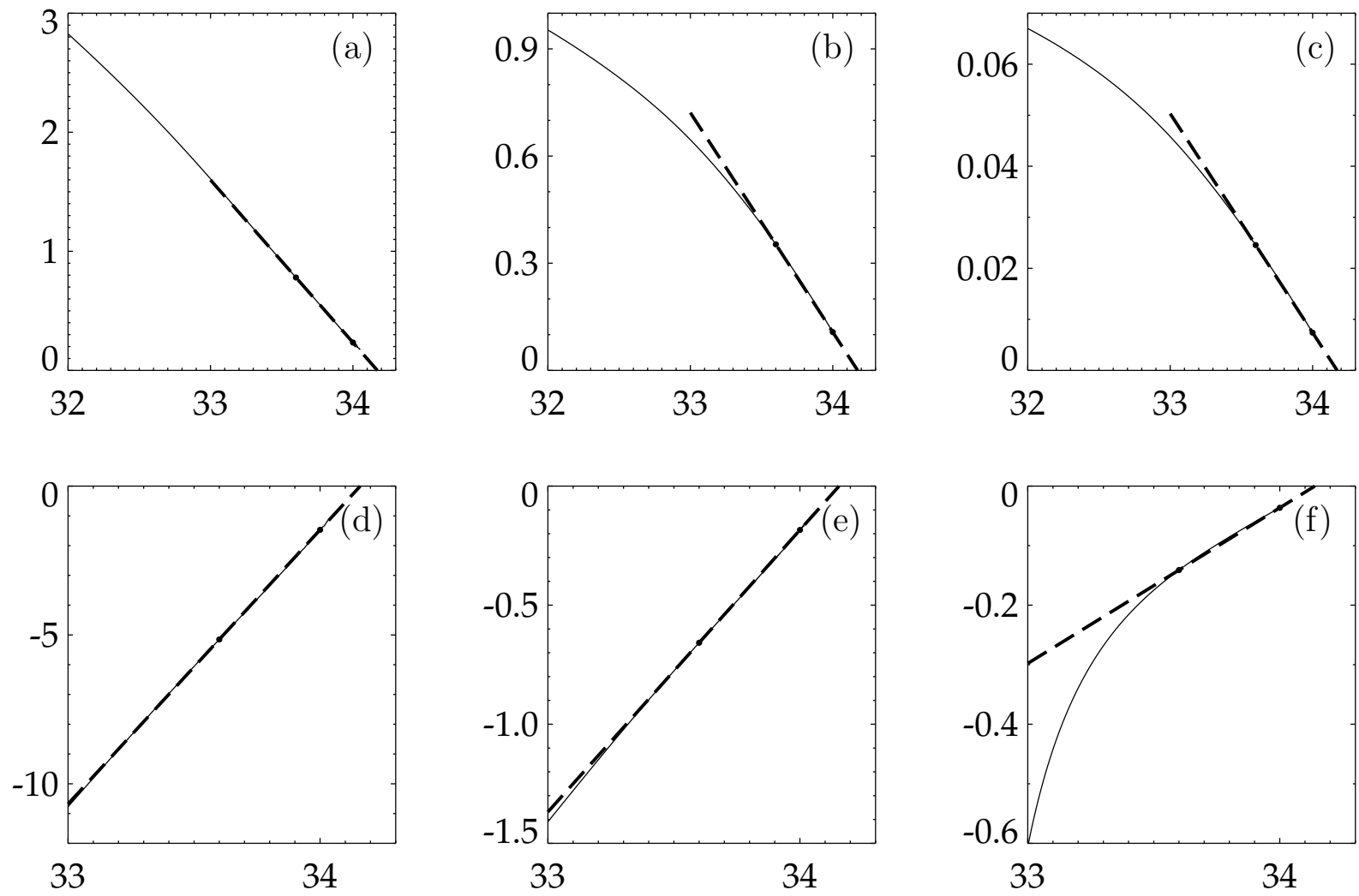

33

34

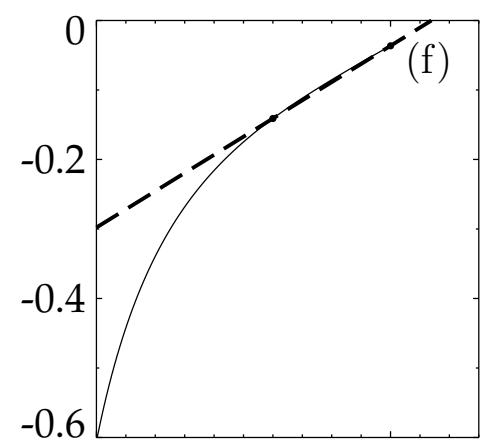

33

34
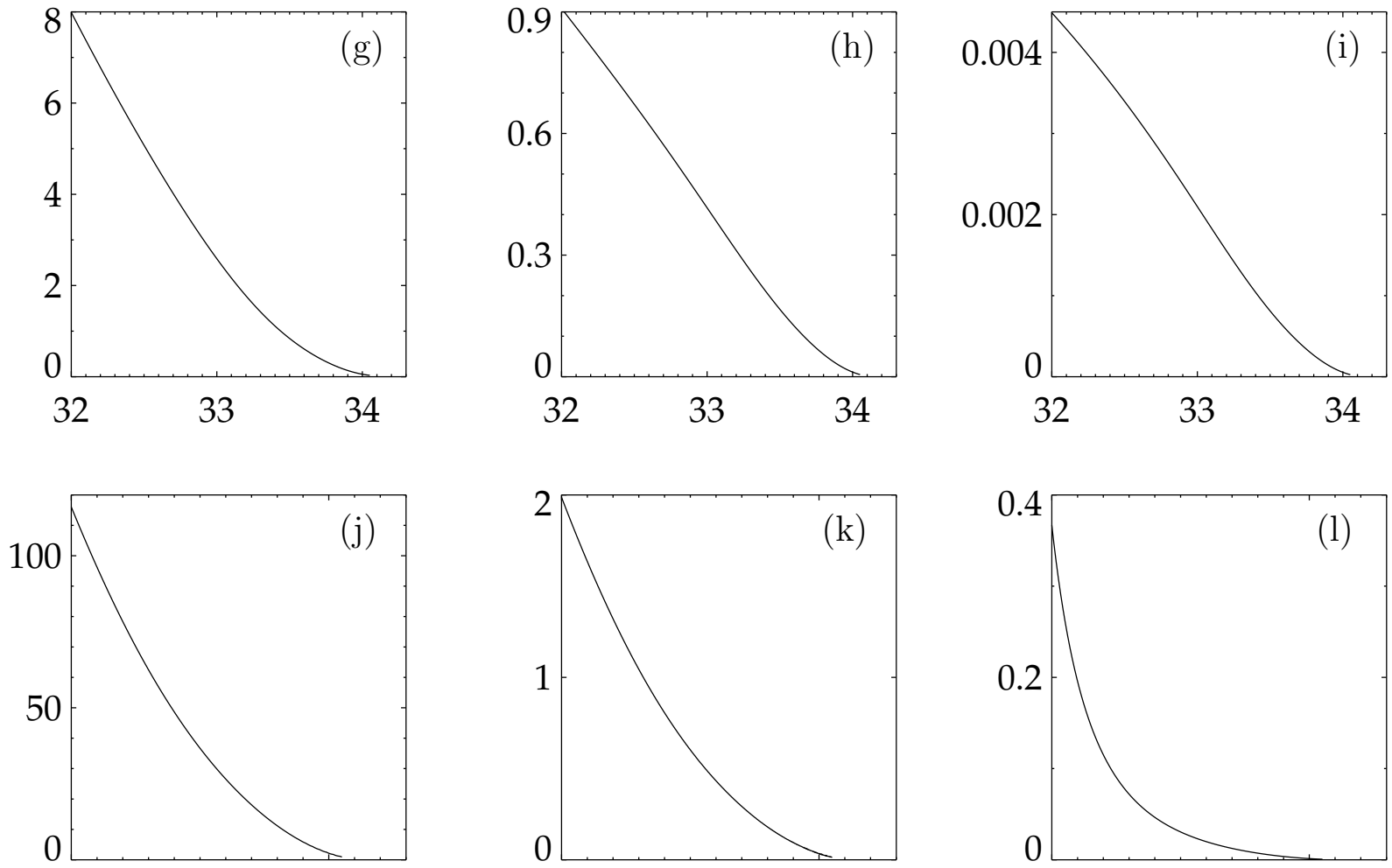

$33 \quad 34$

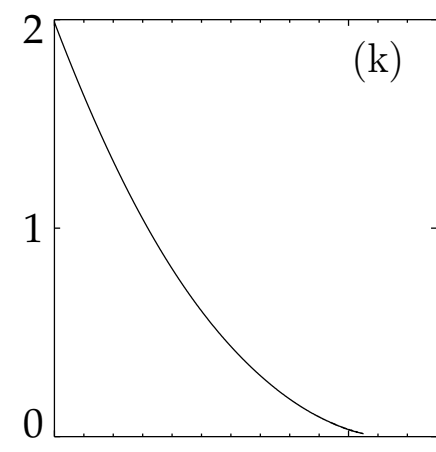

33

34

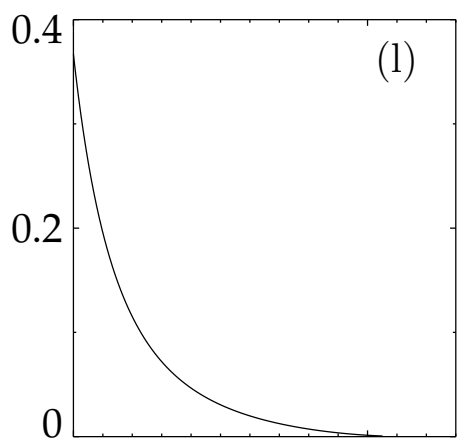

33

34

Figure 3: The quantities (72) (vertical axis) $M_{f, \max }(T)$ (panels (a)-(c)), $M_{f, \min }(T)((\mathrm{d})-(\mathrm{f})), M_{f, \max }^{2}(T)((\mathrm{g})-$ (i)) and $M_{f, \min }^{2}(T)((\mathrm{j})-(\mathrm{l}))$ for $C((\mathrm{a}),(\mathrm{d}),(\mathrm{g}),(\mathrm{j})), c_{03}((\mathrm{~b}),(\mathrm{e}),(\mathrm{h}),(\mathrm{k}))$ and $c_{04}((\mathrm{c}),(\mathrm{f}),(\mathrm{i}),(\mathrm{l}))$ during the preparation of the blow-up of a solution to the system of amplitude equations. Horizontal axis: the slow time $T$. Dashed lines on panels (a)-(f): lines through the solid points on the respective graphs, approximating the asymptotes that characterise the final stage of the process of preparation. 

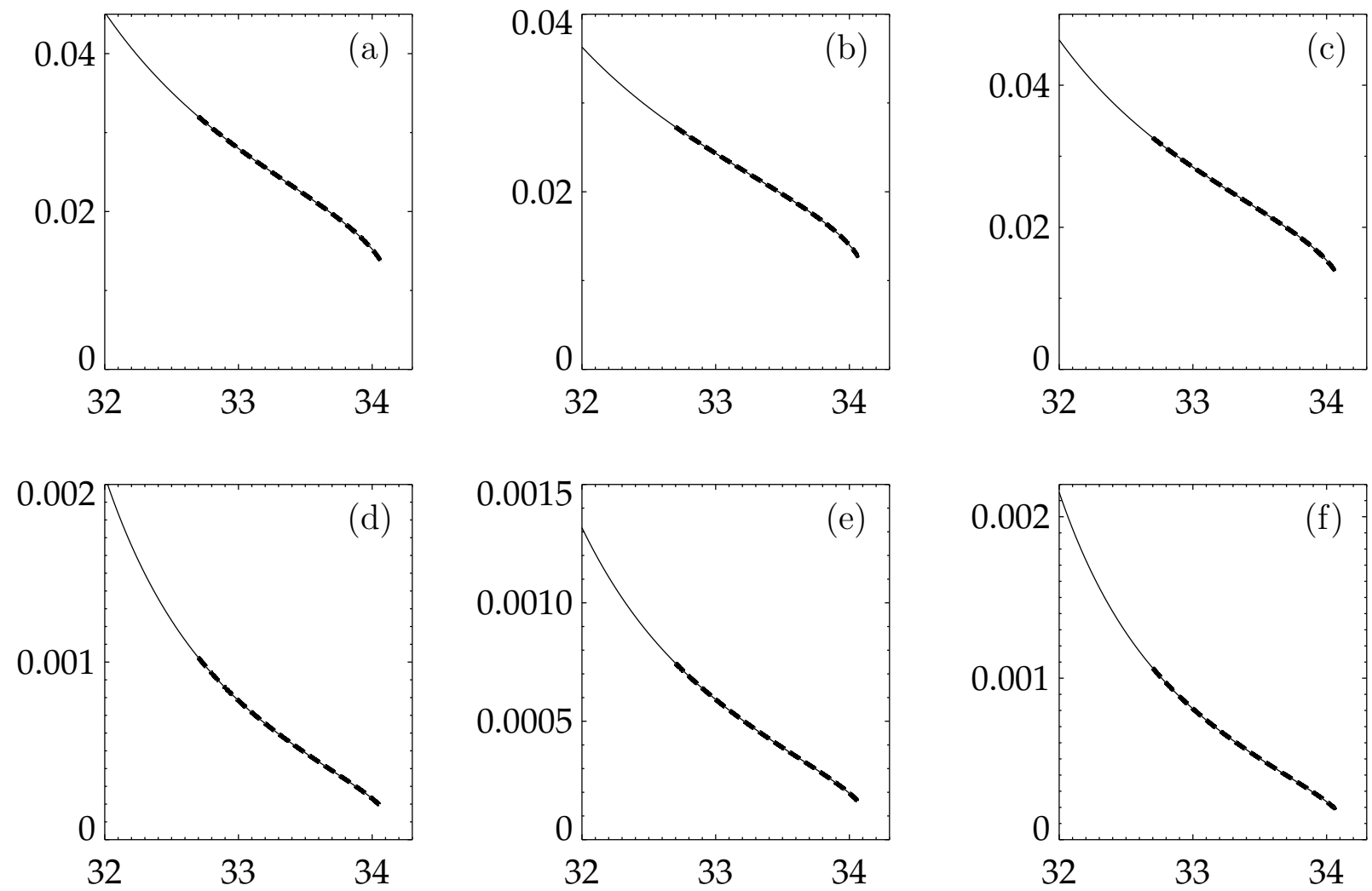

Figure 4: Positions of the maxima (vertical axis), $Q_{f}(T),\left(\right.$ panels (a)-(c)) and $Q_{f}^{2}(T)((\mathrm{d})-(\mathrm{f}))$ for $C((\mathrm{a}),(\mathrm{d})), c_{03}$ $((\mathrm{b}),(\mathrm{e}))$ and $c_{04}((\mathrm{c}),(\mathrm{f}))$ during the preparation of the blow-up of a solution to the system of amplitude equations. Horizontal axis: the slow time $T$. Dashed lines: same quantities computed using a double spatial resolution and a 10 times smaller time step.

of $f(Y, T)$ at time $T$, where as function $f$ we again take the amplitudes $C, c_{03}$ and $c_{04}$. For the self-similar ansatz (71) we would obtain

$$
P_{f}(T)=P_{\Phi}\left(T_{\star}-T\right)^{1 / 2}
$$

where $P_{\Phi}$ is the argument at which the profile $\Phi$ in (71) takes the maximum value; we therefore expect $P_{f}^{2}(T)$ to be a linear function of time. Graphs of positions of the maxima, $P_{f}(T)$, as well as $P_{f}^{2}(T)$ as functions of the slow time $T$ are plotted in Fig. 4, and they do not contradict the hypothesis, that asymptotically (73) holds in the time interval $33 \leq T \leq 34$. However, closer to the time of singularity $T_{\star}$, the graphs of $P_{f}^{2}(T)$ (panels $(\mathrm{d})-(\mathrm{f})$ of Fig. 4) bend down. To check that this bending is genuine and not caused by the lack of resolution, we have recomputed the solution with a twice higher spatial resolution and a 10 times smaller time step; the graphs obtained for the refined positions are shown in Fig. 4 by dashed lines, which visually coincide with the original graphs. Note, that the graphs of $P_{f}^{2}(T)$ (panels (a)-(c) of Fig. 4) feature a similar behaviour, which is close to a linear one at a larger time interval; however, $P_{f}(T)$ bends down near $T_{\star}$ more visibly. Comparison of panels (a)-(c) versus panels (d)-(f) shows, that it is difficult to make a reliable conclusion about the exponent in the argument of $\Phi$ in (73) based on this data.

We have investigated how the occurrence and the time of blow-up depend on the mean amplitudes of the initial conditions (see Fig. 5). The mean amplitudes $\left\langle\left\langle c_{03}\right\rangle\right\rangle$ and $\left\langle\left\langle c_{04}\right\rangle\right\rangle$ of the two neutral zero-mean small-scale stability modes, that exist due to translation invariance in horizontal directions of equations of convective dynamo, appear to be important control parameters: if in the course of temporal evolution these means quit the region of linear stability of constant solutions 


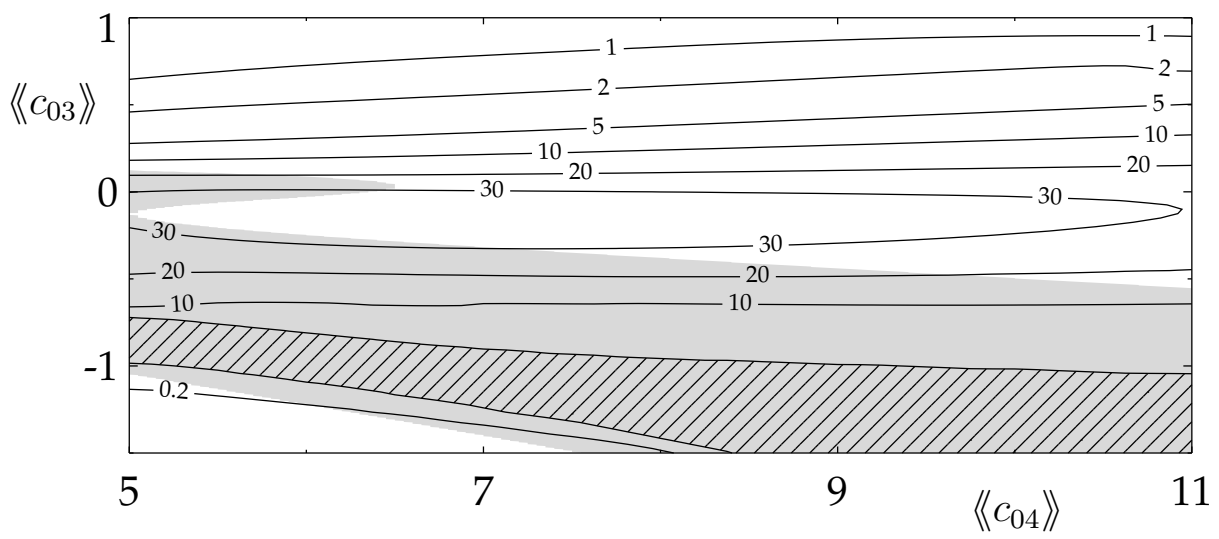

Figure 5: Isolines of blow-up times for varying initial $\left\langle\left\langle c_{03}\right\rangle\right.$ and $\left\langle\left\langle c_{04}\right\rangle\right.$. The region, for which solutions tend to constant values, is cross-hatched. When a point $\left.\left(\left\langle c_{03}\right\rangle\right\rangle,\left\langle c_{04}\right\rangle\right)$ approaches this region, the times of blow-up tend to infinity, and hence there are isolines for the values 10 and higher near the upper border of this region, and for values 1 and higher near the lower border. These isolines are very close to the respective borders and are not shown. The region of linear stability of constant solutions to the system of amplitude equations is shown in gray (cf. Fig. 1).

(see the previous subsection), typically, the solution eventually experiences a blow-up at a finite time.

Ideally, in such an investigation we would like to compare solutions to the system (60) $-(62)$ and (65) obtained for initial conditions where these means are varied and the fluctuating parts are kept unaltered. However, this is impossible, because the initial data must satisfy the non-evolutionary equation (62). Consequently, Fig. 5 is plotted for solutions with the initial conditions constructed as follows: At $T=0$, the fluctuating parts of the amplitudes $c_{03}(Y)$ and $c_{04}(Y)$ are the same in all the runs (they coincide, respectively, with the fluctuating parts of $c_{03}$ and $c_{04}$ in the discussed above main sample run at $T=30$; the r.m.s. means of the fluctuating parts are, respectively, 0.22 and 1.88). The zero-mean amplitude $C(Y)$ is then found from (62) for each pair $\left\langle\left\langle c_{03}\right\rangle\right\rangle,\left\langle\left\langle c_{04}\right\rangle\right\rangle$ separately.

We see in Fig. 5 that the region of mean amplitudes, for which solutions tend to constant values, is within the region of linear stability of constant solutions (see subsection 3.1). We also observe that when a point $\left(\left\langle\left\langle c_{03}\right\rangle\right\rangle,\left\langle\left\langle c_{04}\right\rangle\right\rangle\right)$ approaches the lower boundary of this region outside it, the times of blow-up tend to infinity much faster than when the upper boundary is approached, suggesting that the blow-up preparation proceeds differently, at least in details, in the two areas above and below the region of linear stability of constant solutions. Simulations support this conclusion: For the main sample run, $\left\langle\left\langle c_{03}\right\rangle\right\rangle=0.45,\left\langle\left\langle c_{04}\right\rangle\right\rangle=8.02$ at $T=30$; it corresponds to a point in the area above the region of stability of constant solutions in Fig. 5. An additional run for $\mathrm{Ta}=675$ and the initial conditions, that have been constructed as explained above for $\left\langle\left\langle c_{03}\right\rangle\right\rangle=-1.2$ and $\left\langle\left\langle c_{04}\right\rangle=6\right.$, is represented in Fig. 5 by a point in the area below the region (a video is stored at arXiv.org as an ancillary file Ta675a.mp4). In both runs the graphs of the moduli of Fourier coefficients of the amplitudes versus the wave number $k$ remain near the blow-up predominantly linear. However, while in the main sample run their steepness monotonically decreases in time, in the additional run the steepness experiences a complex oscillatory behaviour. Also, while at the final stage one spike of a large height develops near the time of singularity in the main sample run, high-amplitude spatial oscillations burst out in space in the additional run.

\subsection{Dependence on the Taylor number}

We summarise now the numerical results obtained for several other steady states from the branch $\mathrm{S}_{8}^{\mathrm{R} 1}$. Although the coefficients of the amplitude equations significantly vary within the branch (most of them by more than an order of magnitude), we do not encounter any other types of the temporal behaviour of amplitudes, rather than those found for $\mathrm{Ta}=675$ : the development 

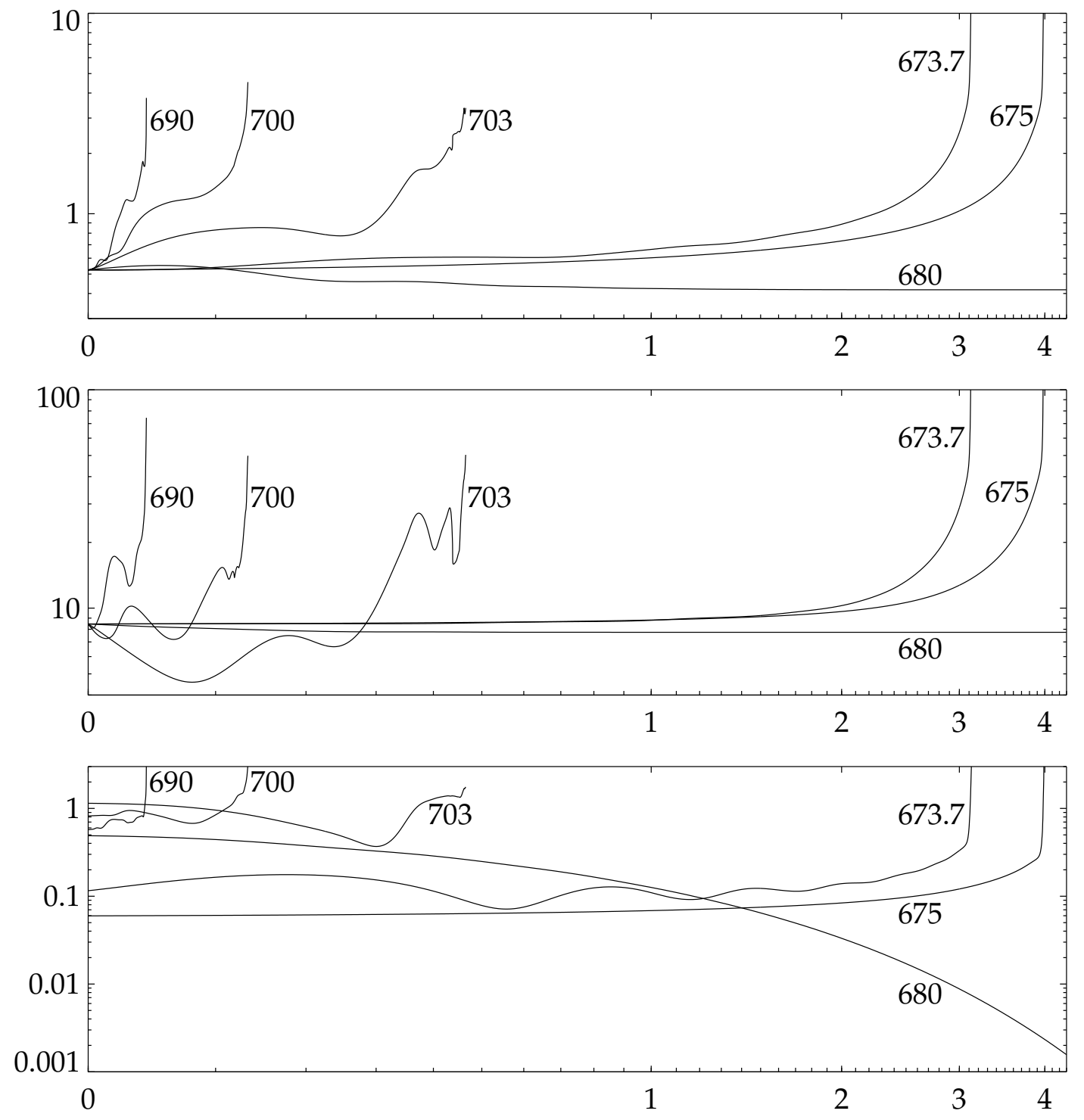

Figure 6: Lebesgue norms (vertical axis) of amplitudes $c_{03}$ (upper panel), $c_{04}$ (middle panel) and $C$ (lower panel) as functions of the slow time $T$ (horizontal axis; the scale is logarithmic to have a better display of details of the graphs for $\mathrm{Ta} \geq 690$, for which the blow-up times are small) for $\mathbf{q}=\mathbf{q}^{1}$ (see Table 2). The plots are labelled by the respective Taylor number values.

of a singularity at a finite time, or the decay of coefficients to constant values, i.e., degeneration of a large-scale perturbation to a neutral zero-mean short-scale mode existing due to the spatial invariance of the convective system.

The genericity of these two types of behaviour is illustrated by Fig. 6, showing the temporal evolution of the Lebesgue norms of amplitudes (defined as $\sqrt{\left\langle\left\langle c^{2}(Y, T)\right\rangle\right.}$ for an amplitude $c(Y, T)$ ) for several values of the Taylor number. The graphs in Fig. 6] are plotted for the initial conditions constructed essentially by the same procedure as in the previous subsection: At $T=0$, amplitudes $c_{03}(Y)$ and $c_{04}(Y)$ are the same in the runs for all considered Ta, and coincide with those in the main sample run at $T=30$. The zero-mean amplitude $C(Y)$ is found from (62) for each Taylor number separately.

Figure 6 shows that the blow-up times depend on the Taylor number non-monotonically. Although for $\mathrm{Ta} \geq 690$ the employed initial profiles of $c_{03}$ and $c_{04}$ quickly lead to a blow-up, for the nearby value $\mathrm{Ta}=680$ the singularity does not develop and the perturbation tends to a short-scale zero-mean neutral mode. Noteworthily, prior to the final stage amplitude $C$ remains close to unity, 
and therefore the cubic nonlinearity of the system of amplitude equations, which is associated with $\xi_{1}=C$ (see (61)), apparently does not play an important role in the development of the singularity.

Further qualitative information is revealed by the videos of the final stage of the preparation of the blow-up in two runs for $\mathrm{Ta}=680$ and 703 (for initial conditions constructed by an alternative procedure), and in the additional run for $\mathrm{Ta}=675$ (in all the three runs $\mathbf{q}=\mathbf{q}^{1}$ ), which are stored at arXiv.org as ancillary files Ta680.mp4, Ta703.mp4 and Ta675a.mp4, respectively. For Ta = 703, like in the runs for $\mathrm{Ta}=675$, the graphs of the moduli of Fourier coefficients of the amplitudes as functions of the wave number $k$ remain predominantly linear, although the fine structure of these graphs is much richer than in the case of the main sample run - all amplitudes have now several extrema, whose magnitudes grow in time. In the main sample run for $\mathrm{Ta}=675$, the steepness of such graphs just decreases in time; by contrast, for $\mathrm{Ta}=703$ initially the steepness increases, so that the round-off level $\sim 10^{-15}$ is attained at $k \sim 60$ (in this simulation, solutions have been truncated to 512 Fourier harmonics in $Y$ after dealiasing), and afterwards the slope starts to decrease; in the additional run for $\mathrm{Ta}=675$ the slope experiences an oscillatory behaviour. Eventually the slope of the graphs decreases until near the time of singularity the graphs become close to horizontal ones. The growth of the extrema (in absolute values) of the amplitudes intensifies in time, but none of the extrema gives rise to a $\delta$-function, as the single maximum of $C$ does in the main sample run for $\mathrm{Ta}=675$. For $\mathrm{Ta}=703$, just before the emergence of the singularity, a secondary fast moving perturbation of amplitudes develops, which modifies their so far preserved forms; the overall linear shape of the graphs of absolute values of the Fourier coefficients becomes deformed. In the additional run for $\mathrm{Ta}=675$, high-frequency high-amplitude spatial oscillations are observed. However, the spatial resolution becoming insufficient, the occurrence of these final events cannot be fully trusted.

The evolution of perturbations near the singularity for $\mathrm{Ta}=680$ significantly differs from those for $\mathrm{Ta}=675$ and 703. The singularity sets in much faster. Two and then three global maxima develop in graphs of the moduli of Fourier coefficients of the amplitudes versus the wave number, which are initially predominantly linear. Each amplitude has initially one maximum; the maxima do not yield $\delta$-functions, but rather burst into packets of amplitude-modulated blobs of high-frequency oscillations.

\section{Concluding remarks}

We have performed a two-scale analysis of large-scale perturbations of a periodic array of steady

thermal convective hydromagnetic states constituting the branch $\mathrm{S}_{8}^{\mathrm{R} 1}$ [7]. These short-scale dynamos are symmetric about the vertical axis and stable to short-scale perturbations. We have shown that, in a collective action, they are capable of generating a large-scale magnetic field, whose geometry is determined by the properties of the short-scale operator of linearisation around the respective steady state. The operation of this large-scale dynamo is based on the joint action of the combined eddy diffusivity and eddy advection, but it does not involve the $\alpha$-effect. To the best of our knowledge, this mechanism is new.

Several remarks are in order.

$1^{\circ}$. We have found that only two small-scale neutral stability modes of a steady convective dynamo with such a group of symmetries have non-vanishing means, each involving a combination of a mean horizontal velocity and magnetic field. The solenoidality in slow variables of the two mean fields implies that only a specific linear combination of these two modes is involved in the asymptotic expansion of a large-scale perturbation, depending on a slow spatial variable and evolving in the slow time. For a given state $\mathbf{W}$ from the considered branch, two such linear combinations can be determined. Two other neutral small-scale stability modes, $\partial \mathbf{W} / \partial x_{i}$, existing due to translation invariance in horizontal directions of equations of convective dynamo, are zero-mean, i.e., their 
amplitudes are not associated with any mean fields. The two amplitudes are essential for the largescale dynamics of the perturbation: if they are both set to zero, then, by virtue of equations (60), the amplitude of the remaining small-scale neutral mode, involving non-zero mean-field components, also vanishes.

This clearly shows that omitting amplitudes of neutral zero-mean small-scale stability modes (when they exist) in the description of the dynamics of multiscale perturbations, which is the common practice in the theory of mean-field electrodynamics, can render the description inadequate. Such modes are not rare; they are featured by translation-invariant and/or time-invariant MHD systems, and can be obtained by differentiating the states, whose stability is analysed, in the spatial variable along the invariant direction and/or time.

$2^{\circ}$. Requirements for the averaging procedures that can be applied also differ significantly between MFE and MST. In MFE, any averaging is deemed acceptable when analysing MHD turbulence, provided it satisfies the Reynolds rules. Accordingly, averaging over a horizontal periodicity cell (or, equivalently, over entire horizontal planes) is often considered in literature. Perhaps, such averaging could be justified in the framework of statistical approach to turbulence (e.g., for ensembles of turbulent eddies). By contrast, our derivation of amplitude equations, following the MST approach and using the PDE homogenisation techniques, shows that the only appropriate averaging when considering two-scale perturbations of laminar MHD regimes is over a periodicity domain of the perturbed state; for time-periodic perturbed states, this must be supplemented by averaging over the temporal period.

Thus, a mathematically rigorous asymptotic procedure leaves no freedom for choosing averaging to our taste; using spatial (or spatio-temporal) averaging is inevitable - this follows from the requirement of solvability of equations in fast variables. Consequently, the MFE approach to such problems (e.g., kinematic dynamo problems concerning generation of large-scale magnetic field by a periodic array of small-scale flow cells) may yield quantitatively incorrect results, when other types of averaging are used, even when they satisfy the Reynolds rules and are allowed in MFE. Furthermore, no conclusions of potential interest for astrophysics can be drawn from the results of [4, 5] on the significant (i.e., responsible for the evolution of large-scale magnetic field) $\alpha$-effect, because averaging over half a periodicity cell performed in these works does not satisfy the Reynolds rules (the significant $\alpha$-effect $i b i d$. is zero).

$3^{\circ}$. The system of amplitude equations that we have derived and studied numerically involves terms describing eddy diffusivity and eddy advection, but not the $\alpha$-effect. This calls for a comparison of the action of these mechanisms of magnetic field generation important in astrophysics.

The spectrum of the $\alpha$-effect operator $\mathbf{H} \mapsto \nabla \times \mathfrak{A H}$, acting, for instance, on space-periodic fields $\mathbf{H}$, is symmetric about the imaginary axis [28] (see also [33], section 3.3.2). Under the action of this operator, a generic perturbation experiences a superexponential growth in slow time, which in this case is $T=\varepsilon t$ (while in the absence of the $\alpha$-effect large-scale amplitudes evolve in $\mathrm{O}(\varepsilon$ ) slower time $T=\varepsilon^{2} t$, see section 2.4). In the MST framework, when $\mathfrak{A} \neq 0$, the $\alpha$-effect operator typically controls the evolution of mean fields of perturbation solely (see [33], sections 3.3.2, 4.2.3, 6.3.2, 7.3.2, 8.3.2). The superexponential growth might be restrained by diffusion, since it is governed by a higher-order differential operator. However, the orders of the $\alpha$-effect and eddy diffusivity differential operators being different, the operators arise in solvability conditions at different orders of the scale ratio and do not coexist. In principle, emergence of the operators of $\alpha$-effect and eddy diffusivity together is not ruled out in the MFE theory, where the so-called $\beta$-effect is sometimes considered, in whose presence the mean e.m.f. becomes

$$
\langle\mathbf{V} \times \mathbf{H}\rangle=\sum_{i, j} \alpha_{i j} H_{j} \mathbf{e}_{i}+\sum_{i, j, k} \beta_{i j k} \frac{\partial H_{j}}{\partial X_{i}} \mathbf{e}_{k}
$$

However, this equality is only partially justified in the MFE theory by considering the truncated 
Taylor expansion of the mean field in the integral operator relating the fluctuating part of the magnetic field to the mean part, and assuming that the respective kernels decay fast (see [22]). Whether this justification can be extended to a formal asymptotic argument is an interesting open question.

Thus, to the best of our knowledge there are no mathematically solid evidences that in multiscale problems, where a rigorous derivation of amplitude equations is possible, the action of the $\alpha$-effect operator can be restrained by eddy diffusivity or the mechanisms of nonlinear saturation. Hence, it can be described as essentially "self-destructing": In a system with the $\alpha$-effect, the perturbation grows superexponentially until it loses the asymptotic smallness and thereby significantly affects the perturbed state. In other words, the $\alpha$-effect can generate magnetic field for a relatively short time, but it destabilises the existing small-scale structure and modifies the entire MHD system into a completely new regime. For this new regime, the analysis of eddy effects must be done anew (provided the scale separation is preserved and the multiscale analysis can be implemented; actually, it is also not guaranteed that the new regime will be capable of generating the field). This is incompatible with the astrophysical reality - large-scale cosmic magnetic fields are known to exist for very long times. Therefore, it is natural to expect that a sequence of such events of self-destruction terminates at an MHD state, where the magnitude of the $\alpha$-effect is sufficiently small not to cause further disturbances.

Within this line of reasoning, the feasibility of the $\alpha$-effect paradoxically, may owe to the phenomenon, originally perceived as significantly reducing the importance of the $\alpha$-effect, namely, the $\alpha$-quenching, i.e., a drastic decrease of the $\alpha$-effect on increasing the magnetic Reynolds number, discovered by S.I. Vainshtein [27]. In the kinematic dynamo problem for a flow with an external scaling, the $\alpha$-effect operator is present in the equation for mean fields together with molecular diffusion [28] (see also [33], ch. 10, 11). In astrophysics, molecular diffusion is weak. However, if the $\alpha$-quenching inhibits the action of the $\alpha$-effect to the levels, where it is essentially offset by molecular magnetic diffusivity, then, at least within this model (also mathematically rigorous), the $\alpha$-effect operator may contribute to stationary magnetic field generation without causing the restructuring of the MHD system hosting it.

As our results show (see section 3), in the absence of the $\alpha$-effect the overall character of the large-scale evolution is not that different. If negative combined eddy diffusivity is present, then a generic perturbation grows superexponentially. As mentioned above, the characteristic slow time of the large-scale evolution is then slower than for the $\alpha$-effect, but the ultimate result of the evolution is the same: destruction of the underlying MHD regime and the onset of a new one (which can lack scale separation). We have no evidence that taking into account nonlinear terms in equations for weakly nonlinear perturbations can halt such processes. Here we have studied numerically the case, where the action of the combined eddy diffusivity is stabilising (as that of molecular diffusivity). In this case the large-scale evolution significantly depends on how large is the initial perturbation. When it is below a certain threshold, the amplitudes tend to constants and the perturbation evolves to a linear combination of the two zero-mean neutral modes; otherwise, it blows up in apparently a finite time. Therefore, like in the presence of the $\alpha$-effect, the large-scale evolution controlled by eddy diffusivity and eddy advection can perturb the original small-scale dynamics till its structure is significantly modified, but the growth rate of these processes is much, $\mathrm{O}(\varepsilon)$, slower.

An open question is whether the system of amplitude equations (60)-(62) and (65) has a solution which is a travelling wave. We have failed to find this regime for the steady dynamo at Ta $=675$, but this can be due to the scarceness of our efforts; also, it can, in principle, exist for perturbations of other steady states from the branch that we have considered, or in other branches with the symmetries compatible with our multiscale analysis (such as the time-periodic dynamo $\mathrm{P}_{4}^{\mathrm{R} 1}$ ). If such a solution exists and is stable, it would be an example of a large-scale perturbation that does not act self-destructively as just discussed; if it is unstable in slow variables, it is nevertheless of 
interest as an invariant object in the phase space influencing the dynamics of amplitudes.

$4^{\circ}$. We have analysed numerically the system of amplitude equations derived here for convective hydromagnetic regimes with the symmetry group of states in the branch $\mathrm{S}_{8}^{\mathrm{R} 1}$. The behaviour of its solutions is complex, because this is a mixed system, involving both evolutionary and nonevolutionary nonlinear equations. Many questions remain open, for instance: Does this system allow patterns of behaviour of perturbation, different from those that we have identified? Does the blow-up occur for other boundary conditions? Does the solution for Ta $=675$ "converge" at the blow-up time to a distribution involving the $\delta$-function, as computations suggest? If this $\delta$-function is interpreted as a derivative of certain quantities experiencing a jump, can these quantities be identified in physical terms? How can a solution be continued in some weak sense beyond the blow-up time $T_{\star}$ ?

$5^{\circ}$. The system of amplitude equations that we have derived is very restrictive: it can only be used to describe weakly nonlinear perturbations, where amplitudes are initially constant on lines, perpendicular to certain well-defined directions $\mathbf{q}$ on the plane of slow variables. This system, therefore, does not describe general two-scale perturbations of a convective MHD state, that are of the form (12)-(14) and obey equations (8)-(11). An interesting open question is to construct an asymptotic formalism for perturbations of a more general form.

$6^{\circ}$. Finally, it is of interest to perform similar investigations for other branches of short-scale convective hydromagnetic regimes: (i) for time-periodic MHD states constituting branch $\mathrm{P}_{4}^{\mathrm{R} 1}$ that emerge in the interval $704<\mathrm{Ta}<705$ in a Hopf bifurcation from $\mathrm{S}_{8}^{\mathrm{R} 1}$ considered here (largescale perturbations of time-periodic states involve an additional amplitude); (ii) for steady states and time-periodic regimes constituting the branches, found in [7], that possess other groups of symmetries, for which $\mathcal{U}=0$.

\section{Acknowledgments}

We are grateful to Erico Rempel for discussions. RC acknowledges financial support from FAPESP (Brazil, grant 2013/01242-8).

\section{References}

[1] Baptista M., Gama S.M.A., Zheligovsky V. Eddy diffusivity in convective hydromagnetic systems. Eur. Phys. J. B. 60, 337-352 (2007).

[2] Barenblatt G.I. Scaling, self-similarity, and intermediate asymptotics. Cambridge Univ. Press, 1996.

[3] Braginsky S.I. Self-excitation of a magnetic field during the motion of a highly conducting fluid. J. Exper. Theor. Phys. 47, 1084-1098 (1964). Engl. transl. Sov. Phys. J.E.T.P. 20, 726-735 (1965).

[4] Cattaneo F., Hughes D.W. Dynamo action in a rotating convective layer. J. Fluid Mech. 553, 401-418 (2006).

[5] Cattaneo F., Hughes D.W. The alpha-effect in rotating convection: size matters. J. Fluid Mech. 594, 445-461 (2008).

[6] S. Chandrasekhar. Hydrodynamic and hydromagnetic stability. Oxford Univ. Press, 1961.

[7] Chertovskih R., Gama S.M.A., Podvigina O., Zheligovsky V. Dependence of magnetic field generation by thermal convection on the rotation rate: a case study. Physica D, 239, 11881209 (2010). 
[8] Dubrulle B., Frisch U. Eddy viscosity of parity-invariant flow. Phys. Rev. A. 43, 5355-5364 (1991).

[9] Eggers J., Fontelos M.A. The role of self-similarity in singularities of partial differential equations. Nonlinearity, 22, R1-R44 (2009).

[10] Fokkema D.R. Subroutine BISTBL. 1995. http://www.staff.science.uu.nl/ vorst102/ software.html

[11] Gama S., Vergassola M., Frisch U. Negative eddy viscosity in isotropically forced twodimensional flow: linear and nonlinear dynamics. J. Fluid Mech. 260, 95-126 (1994).

[12] D. Gottlieb, S.A. Orszag. Numerical analysis of spectral methods: theory and applications. CBMS-NSF regional conference series in applied mathematics (vol. 26). SIAM, 1977.

[13] Krause F., Rädler K.-H. Mean-field magnetohydrodynamics and dynamo theory. AcademicVerlag, Berlin, 1980.

[14] Lanotte A., Noullez A., Vergassola M., Wirth A. Large-scale dynamo by negative magnetic eddy diffusivities. Geophys. Astrophys. Fluid Dyn. 91, 131-146 (1999).

[15] Larmor J. How could a rotating body such as the Sun become a magnet? Rep. Brit. Assoc. Adv. Sci. 159-160 (1919).

[16] Larmor J. Possible rotational origin of magnetic fields of Sun and Earth. Electr. Rev. 85, 412 (1919).

[17] S.A. Orszag. On the elimination of aliasing in finite-difference schemes by filtering highwavenumber components. J. Atmos. Sci. 28, 1074 (1971).

[18] Parker E.N. The solar hydrodynamic dynamo. Proc. Nat. Acad. Sci. US. 43, 8-13 (1957).

[19] Parker E.N. Magnetic fields in the cosmos. Sci. Amer. 249, 36-46 (1983).

[20] Polyanin A.D., Zaitsev V.F. Handbook of nonlinear partial differential equations. Taylor \& Francis Group, Boca Raton, 2012. 2nd ed.

[21] Press W.H., Teukolsky S.A., Vetterling W.T., Flannery B.P. Numerical recipes in Fortran. The art of scientific computing. Cambridge Univ. Press, 1992. 2nd ed.

[22] Rädler K.-H. Mean-field dynamo theory: early ideas and today's problems. In Magnetohydrodynamics. Historical evolution and trends. Eds. Molokov S., Moreau R., Moffatt K. Fluid mechanics and its applications, vol. 80. Springer, 2007, 55-72.

[23] Sleijpen G.L.G., Fokkema D.R. BiCGstab( $\ell)$ for linear equations involving unsymmetric matrices with complex spectrum. ETNA, 1, 11-32 (1993).

[24] Sleijpen G.L.G., van der Vorst H.A. Maintaining convergence properties of BiCGstab methods in finite precision arithmetic. Numerical algorithms, 10, 203-223 (1995).

[25] Sleijpen G.L.G., van der Vorst H.A. Reliable updated residuals in hybrid BiCG methods. Computing, 56, 141-163 (1996). 
[26] Steenbeck M., Krause F., Rädler K.-H. Berechnung der mittleren Lorentz-Feldstärke $\overline{\boldsymbol{v} \times \boldsymbol{b}}$ für ein elektrisch leitendes Medium in turbulenter, durch Coriolis-Kräfte beeinfluißter Bewegung. Z. Naturforsch. 21a, 369-376 (1966). Engl. transl.: A calculation of the mean electromotive force in an electrically conducting fluid in turbulent motion, under the influence of Coriolis forces. In Roberts P.H., Stix M. The turbulent dynamo: A translation of a series of papers by F. Krause, K.-H. Rädler, and M. Steenbeck. Tech. Note NCAR-TN/IA-60, Boulder, Colorado, 1971, 29-47. http://nldr. library.ucar.edu/repository/assets/technotes/TECH-NOTE-000-000-000-045.pdf .

[27] Vainshtein S.I., Cattaneo F. Nonlinear restrictions on dynamo action. Astrophys. J. 393, 165171 (1992).

[28] Vishik M.M. Periodic dynamo. In Mathematical methods in seismology and geodynamics (Computational seismology. 19), 186-215. Eds. Keilis-Borok V.I., Levshin A.L. Nauka, Moscow, 1986. Engl. transl.: Computational seismology. 19, 176-209. Allerton Press, New York, 1987.

[29] Zheligovsky V.A. Weakly nonlinear stability of magnetohydrodynamic systems with a center of symmetry to perturbations involving large scales. Fiz. Zemli. № 3, 69-78 (2006). Engl. transl.: Zheligovsky V.A. Weakly nonlinear stability of centrally symmetric magnetohydrodynamic systems to perturbations involving large scales. Izvestiya, Physics of the Solid Earth. 42, 244$253(2006)$.

[30] Zheligovsky V.A. Mean-field equations for weakly non-linear multiscale perturbations of forced hydromagnetic convection in a rotating layer. Geophys. Astrophys. Fluid Dyn. 102, 489-540 (2008).

[31] Zheligovsky V. Amplitude equations for weakly nonlinear two-scale perturbations of free hydromagnetic convective regimes in a rotating layer. Geophys. Astrophys. Fluid Dyn. 103, 397-420 (2009).

[32] Zheligovsky V. Generation of a symmetric magnetic field by thermal convection in a plane rotating layer. Magnetohydrodynamics, 46, 3-22 (2010).

[33] Zheligovsky V.A. Large-scale perturbations of magnetohydrodynamic regimes: linear and weakly nonlinear stability theory. Lecture Notes in Physics, vol. 829, Springer-Verlag, Heidelberg, 2011.

[34] Zheligovsky V.A., Podvigina O.M. Generation of multiscale magnetic field by parity-invariant time-periodic flows. Geophys. Astrophys. Fluid Dyn. 97, 225-248 (2003).

[35] Zheligovsky V.A., Podvigina O.M., Frisch U. Dynamo effect in parity-invariant flow with large and moderate separation of scales. Geophys. Astrophys. Fluid Dyn. 95, 227-268 (2001). 